https://doi.org/10.15407/ujpe63.10.863

K.A. BUGAEV,${ }^{1}$ A.I. IVANYTSKYI, ${ }^{2,1}$ V.V. SAGUN,${ }^{1,3}$ E.G. NIKONOV,${ }^{4}$ G.M. ZINOVJEV ${ }^{1}$

${ }^{1}$ Bogolyubov Institute for Theoretical Physics, Nat. Acad. of Sci. of Ukraine (14b, Metrolohichna Str., Kyiv 03680, Ukraine; e-mail: bugaev@th.physik.uni-frankfurt.de, Gennady.Zinovjev@cern.ch)

2 Department of Fundamental Physics, University of Salamanca

(Plaza de la Merced s/n 37008, Spain; e-mail: oivanytskyi@usal.es)

${ }^{3}$ CENTRA, Instituto Superior Técnico, Universidade de Lisboa

(Av. Rovisco Pais 1, 1049-001 Lisboa, Portugal; e-mail: v_sagun@ukr.net)

${ }^{4}$ Laboratory for Information Technologies, JINR

(6, Joliot-Curie Str., Dubna 141980,Russia; e-mail: e.nikonov@jinr.ru)

\title{
EQUATION OF STATE OF QUANTUM GASES BEYOND THE VAN DER WAALS APPROXIMATION
}

\begin{abstract}
$A$ recently suggested equation of state with the induced surface tension is generalized to the case of quantum gases with mean-field interaction. The self-consistency conditions of such a model and the conditions necessary for the Third Law of thermodynamics to be satisfied are found. The quantum virial expansion of the van der Waals models of such a type is analyzed, and its virial coefficients are given. In contrast to traditional beliefs, it is shown that an inclusion of the third and higher virial coefficients of a gas of hard spheres into the interaction pressure of the van der Waals models either breaks down the Third Law of thermodynamics or does not allow one to go beyond the van der Waals approximation at low temperatures. It is demonstrated that the generalized equation of state with the induced surface tension allows one to avoid such problems and to safely go beyond the van der Waals approximation. In addition, the effective virial expansion for the quantum version of the induced surface tension equation of state is established, and all corresponding virial coefficients are found exactly. The explicit expressions for the true quantum virial coefficients of an arbitrary order of this equation of state are given in the low-density approximation. A few basic constraints on such models which are necessary to describe the nuclear and hadronic matter properties are discussed.
\end{abstract}

Ke ywords: nuclear matter, hadron resonance gas, induced surface tension, quantum gases, virial coefficients.

\section{Introduction}

Investigation of the equation of state (EoS) of strongly interacting particles at low temperatures is important for studies of the nuclear liquid-gas phase transition and for properties of neutron stars [13]. To have a realistic EoS, one has to simultane-

(C) K.A. BUGAEV, A.I. IVANYTSKYI, V.V. SAGUN, E.G. NIKONOV, G.M. ZINOVJEV, 2018

ISSN 2071-0194. Ukr. J. Phys. 2018. Vol. 63, No. 10 ously account for a short-range repulsive interaction, a medium-range attraction, and the quantum properties of particles. Unfortunately, it is not much known about the in-medium quantum distribution functions of particles which experience a strong interaction. Therefore, a working compromise to account for all these features is to introduce the quasiparticles with quantum properties which interact via the mean field. One of the first successful models of such a type 
was a Walecka model [4]. However, the strong demands to consider a more realistic interaction which is not restricted by some kind of effective Lagrangian led to formulating a few phenomenological generalizations of the relativistic mean-field model [5-7]. Although a true breakthrough among them was made in work [7] in which the hard-core repulsion was suggested for fermions, the introduction of a phenomenological attraction in the spirit of the Skyrme-HartreeFock approach $[8,9]$ which depends not on the scalar field, but on the baryonic charge density, was also important, since such a dependence of the attractive mean-field is typical of the EoS of real gases [10].

However, in addition to the usual defect of the relativistic mean-field models breaking down the first and second Van Hove axioms of statistical mechanics $[11,12]$, the usage of a non-native variable, namely a particle number density, in the grand canonical ensemble led to the formulation of self-consistency conditions $[5,6]$. In contrast to the Walecka model [4] and its followers for which the structure of a Lagrangian and the extremum condition of the system pressure with respect to each mean-field automatically provide the fulfillment of the thermodynamic identities, the phenomenological mean-field EoS of hadronic matter had to be supplemented by the self-consistency conditions $[5,6,13]$. The latter allows one to, formally, recover the first axiom of statistical mechanics $[11,12]$ (for the more recent discussion of the self-consistency conditions, see [1416]). An exception is given by the van der Waals (VdW) hard-core repulsion [7], since such an interaction in the grand canonical ensemble depends on the system pressure which is the native variable for it.

Due to its simplicity, the VdW repulsion is very popular in various branches of modern physics. But even in case of Boltzmann statistics, it is valid only at low particle densities for which an inclusion of the second virial coefficient is sufficient. For the classical gases, the realistic EoSs which are able to account for several virial coefficients are well-known [10, 17], while a complete quantum mechanical treatment of the third and higher virial coefficients is rather hard [18]. Hence, the quantum EoSs with realistic interaction allowing one to go beyond the second virial coefficient are of great interest not only for the dense hadronic and nuclear/neutron systems, but also for quantum and classical liquids. It is widely believed that one possible way to go beyond the VdW approximation, i.e. beyond the second virial coefficient, is to include a sophisticated interaction known from the classical models $[10,17]$ into the relativistic meanfield models with the quantum distribution functions for quasiparticles $[14,15]$.

On the other hand, a great success in getting a high quality description of experimental hadronic multiplicities measured in the central nuclear collisions from AGS (BNL) to LHC (CERN) energies is achieved recently with the hadron resonance gas model which employs both the traditional VdW repulsion [19-24] and the induced surface tension (IST) concept for the hard-core repulsion [25-27] motivates us to formulate and to throughly inspect the quantum version of this novel class of the IST EoSs in order to apply it in the future to the description of the properties of dense hadronic, nuclear, neutron matter and dense quantum liquids on the same footing. This is a natural choice, since the Boltzmann version of the IST EoS [25, 26] for a single sort of particles simultaneously accounts for the second, third, and fourth virial coefficients of the classical gas of hard spheres and, thus, allows one to go beyond the VdW approximation. The multicomponent formulation of such an EoS applied to a mixture of nuclear fragments with all possible sizes [28] not only allows one to introduce the compressibility of atomic nuclei into an exactly solvable version [29] of the statistical nuclear multifragmentation model [30], but also it sheds light on the reason of why this model employing the proper volume approximation for the hard-core repulsion is able to correctly reproduce the low-density virial expansion for all atomic nuclei.

Therefore, the present work has two aims. First, we would like to analyze the popular quantum VdW models [14-16] at high and low temperatures in order to verify whether a tuning of the interaction allows one to go beyond the VdW treatment. In addition, we calculate all virial coefficients for the pressure of point-like particles of the quantum VdW EoS. Second, we generalize the recently suggested IST EoS $[25,26]$ to the quantum case, obtain its effective virial expansion, and calculate all quantum virial coefficients, including the true virial coefficients for the low-density limit. Using these results, we discuss a few basic constraints on the quantum EoS which are necessary to model the properties of nuclear/neutron and hadronic matter.

ISSN 2071-0194. Ukr. J. Phys. 2018. Vol. 63, No. 10 
The work is organized as follows. In Sect. 2 we analyze, the quantum VdW EoS and its virial expansion and discuss the pitfalls of this EoS. The quantum version of the IST EoS is suggested and analyzed in Sect. 3. In Sect. 4, we obtain several virial expansions of this model and discuss the Third Law of thermodynamics for the IST EoS. Some simplest applications to nuclear and hadronic matter EoS are discussed in Sect. 5, while our conclusions are formulated in Sect. 6.

\section{Quantum Virial}

\section{Expansion for the VdW Quasiparticles}

Similarly to the ordinary gases, the source of hardcore repulsion in the hadronic or nuclear systems is related to the Pauli blocking effect between the interacting fermionic constituents existing inside of the composite particles (see, e.g., [2]). This effect appears due to the requirement of antisymmetrization of the wave function of all fermionic constituents existing in the system. At very high densities, it may lead to the Mott effect, i.e., to the dissociation of composite particles or even the clusters of particles into their constituents [2]. Therefore, it is evident that, at sufficiently high densities, one cannot ignore the hardcore repulsion or the finite (effective) size of composite particles. The success of traditional EoSs used in the theory of real gases [10] based on the hardcore repulsion approach tells us that this is a fruitful framework also for quantum systems. Hence, we start from the simplest case, i.e., the quantum VdW EoS $[15,16]$. The typical form of EoS for quantum quasiparticles of mass $m_{p}$ and degeneracy factor $d_{p}$ is as follows:

$p\left(T, \mu, n_{i d}\right)=p_{i d}\left(T, \nu\left(\mu, n_{i d}\right)\right)-P_{\text {int }}\left(T, n_{i d}\right)$,

$p_{i d}(T, \nu)=d_{p} \int \frac{d \mathbf{k}}{\left(2 \pi^{3}\right)} \frac{k^{2}}{3 E(k)} \frac{1}{e^{\left(\frac{E(k)-\nu}{T}\right)}+\zeta}$,

$\nu\left(\mu, n_{i d}\right)=\mu-b p+U\left(T, n_{i d}\right)$,

where the constant $b \equiv 4 V_{0}=\frac{16 \pi}{3} R_{p}^{3}$ is the excluded volume of particles with the hard-core radius $R_{p}$ (here, $V_{0}$ is their proper volume), the relativistic energy of particle with momentum $\mathbf{k}$ is $E(k) \equiv$ $\equiv \sqrt{\mathbf{k}^{2}+\mathbf{m}_{p}^{2}}$, and the density of point-like particles is defined as $n_{i d}(T, \nu) \equiv \frac{\partial p_{i d}(T, \nu)}{\partial \nu}$. The parameter $\zeta$ switches between the Fermi $(\zeta=1)$, Bose $(\zeta=-1)$, and Boltzmann $(\zeta=0)$ statistics. The interaction part of the pressure $P_{\text {int }}\left(T, n_{i d}\right)$ and the mean-field potential $U\left(T, n_{i d}\right)$ will be specified later.

Note that, similarly to the Skyrme-like EoS and the EoS of real gases, it is assumed that the interaction between quasiparticles described by system (1)-(3) is completely accounted by the excluded volume (hardcore repulsion), by the mean-field potential $U\left(T, n_{i d}\right)$, and by the pressure $P_{\text {int }}\left(T, n_{i d}\right)$. This is in contrast to the relativistic mean-field models of the Walecka type in which the mass shift of quasiparticles is taken into account. Since such an effect may be important for the modeling of the chiral symmetry restoration in hadronic matter (the strongest arguments of its existence are recently given in [27]), we leave it for a future exploration and concentrate here on a simpler EoS defined by Eqs. (1)-(3).

The functions $U\left(T, n_{i d}\right)$ and $P_{\text {int }}\left(T, n_{i d}\right)$ are not independent, due to the thermodynamic identity $n\left(T, \nu\left(\mu, n_{i d}\right)\right) \equiv \frac{\partial p\left(T, \nu\left(\mu, n_{i d}\right)\right)}{\partial \mu}$. Therefore, the meanfield terms $U$ and $\stackrel{\partial \mu}{P_{\text {int }}}$ should obey the selfconsistency condition

$n_{i d} \frac{\partial U\left(T, n_{i d}\right)}{\partial n_{i d}}=\frac{\partial P_{\text {int }}\left(T, n_{i d}\right)}{\partial n_{i d}} \Rightarrow$
$\Rightarrow P_{\text {int }}\left(T, n_{i d}\right)=n_{i d} U\left(T, n_{i d}\right)-\int_{0}^{n_{i d}} d n U(T, n)$.

After integrating by parts Eq. (4), we used the obvious condition $U(T, 0)<\infty$ in (5). If condition (5) is obeyed, then the direct calculation of the $\mu$-derivative of the pressure (1) gives the usual expression for the particle number density in terms of the density of point-like particles

$n=\frac{n_{i d}}{1+b n_{i d}}$

$n_{i d}(T, \nu)=d_{p} \int \frac{d \mathbf{k}}{\left(2 \pi^{3}\right)} \frac{1}{e^{\left(\frac{E(k)-\nu}{T}\right)}+\zeta}$.

From these equations, one finds that $n \rightarrow b^{-1}$ for $n_{i d} \rightarrow \infty$. The limit $n_{i d} \rightarrow \infty$ is provided by the condition $\nu \rightarrow \infty$ or $T \rightarrow \infty$ for $\zeta=\{0 ; 1\}$, while, for $\zeta=-1$, it is provided by the condition $\nu \rightarrow m_{p}-0$ or $T \rightarrow \infty$.

Note that, in contrast to other works discussing Eqs. (4) and (5), we will use the density of pointlike particles $n_{i d}$ through this paper as an argument 
of the functions $U\left(T, n_{i d}\right)$ and $P_{\text {int }}\left(T, n_{i d}\right)$ instead of the physical density of particles $n$, because, for more sophisticated EoSs, their relation will be more complicated than (6). In addition, such a representation is convenient for a subsequent analysis, because the virial expansion of $p_{i d}(T, \nu)$ in terms of $n_{i d}(T, \nu)$ looks extremely simple [18]:

$p_{i d}(T, \nu)=T \sum_{l=1}^{\infty} a_{l}^{(0)}\left[n_{i d}(T, \nu)\right]^{l}$,

where

$a_{1}^{(0)}=1$,

$a_{2}^{(0)}=-b_{2}^{(0)}$,

$a_{3}^{(0)}=4\left[b_{2}^{(0)}\right]^{2}-2 b_{3}^{(0)}$,

$a_{4}^{(0)}=-20\left[b_{2}^{(0)}\right]^{3}+18 b_{2}^{(0)} b_{3}^{(0)}-3 b_{4}^{(0)}$,

Here, the first several virial coefficients $a_{l}^{(0)}$ of an ideal quantum gas are expressed in terms of the corresponding cluster integrals $b_{l>1}^{(0)}$ which depend only on the temperature. The latter can be expressed via the thermal density of the auxiliary Boltzmann system $\left.n_{i d}^{(0)}(T, \nu) \equiv n_{i d}(T, \nu)\right|_{\zeta=0}$ of Eq. (7) $[18,31]$

$b_{l}^{(0)}=\frac{(\mp 1)^{l+1}}{l} n_{i d}^{(0)}(T / l, \nu)\left[n_{i d}^{(0)}(T, \nu)\right]^{-l}$,

where the upper (lower) sign corresponds to Fermi (Bose) statistics. For the non-relativistic case, expression (14) can be further simplified [18]. For an arbitrary degeneracy factor $d_{p}$, it acquires the form [31]

$\left.b_{l}^{(0)}\right|_{\text {nonrel }} \simeq \frac{(\mp 1)^{l+1}}{l^{\frac{5}{2}}}\left(\frac{1}{d_{p}}\left[\frac{2 \pi}{T m_{p}}\right]^{\frac{3}{2}}\right)^{l-1}$.

For high temperatures, one can write an ultra-relativistic analog of Eq. (15) for a few values of $l=$ $=2,3, \ldots \ll T / m_{p}$

$\left.b_{l}^{(0)}\right|_{\mathrm{urel}} \simeq \frac{(\mp 1)^{l+1}}{l^{4}}\left[\frac{\pi^{2}}{d_{p} T^{3}}\right]^{l-1}$.

Suppose that the coefficients $a_{l}^{(0)}$ from Eq. (8) are known and that the virial expansion is convergent for the considered $T$. Then, using Eq. (6), we find $n_{i d}=n /(1-b n)$. Hence, we can rewrite Eq. (8) as

$\frac{p_{i d}(T, \nu)}{T n}=\frac{1}{1-b n}+\sum_{l=2}^{\infty} a_{l}^{(0)} \frac{[n]^{l-1}}{[1-b n]^{l}}$.

Note that the expansions of such a type for a system pressure which use the variable $n /(1-b n)$ instead of $n$ are well-known for the EoSs of hard discs [32] and hard spheres [33], since they provide a very fast convergence of the series due to a very fast decrease of their coefficients.

As one can see from Eqs. (15) and (16), at high temperatures, all cluster integrals and virial coefficients of the ideal quantum gas strongly decrease with the temperature $T$ and, hence, at high temperatures, the virial expansion of $p_{i d}(T, \nu)$ is defined by the first (classical) term on the right-hand side of (17). In this case, one gets

$\frac{p_{i d}(T, \nu)}{T n} \simeq 1+4 V_{0} n+\left(4 V_{0} n\right)^{2}+\left(4 V_{0} n\right)^{3}+\ldots$.

Here, after expanding the first term on the righthand side of (17), we used the relation between $b$ and $V_{0}$. From this equation, one sees that only the second virial coefficient, $4 V_{0}$, coincides with the one for the gas of hard spheres, while the third, $16 V_{0}^{2}$, and the fourth, $64 V_{0}^{3}$ virial coefficients are essentially larger than their counterparts $B_{3}=10 V_{0}^{2}$ and $B_{4}=18.36 V_{0}^{3}$ of the gas of hard spheres. In addition, Eq. (17) can naturally explain why the authors of work [14] insisted on the interaction pressure $P_{\text {int }}$ to be a linear function of $T$ (see a statement after Eq. (62) in [14]): if one chooses the interaction pressure in the form

$P_{\text {int }}\left(T, n\left(n_{i d}\right)\right)=T F\left(n\left(n_{i d}\right)\right)=T n \times$
$\times\left[\left(b^{2}-B_{3}\right) n^{2}+\left(b^{3}-B_{4}\right) n^{3}+\left(b^{4}-B_{5}\right) n^{4}+\ldots\right]$,

then, at high temperatures, the quantum corrections are negligible. Hence, for such a choice of $P_{\text {int }}\left(T, n\left(n_{i d}\right)\right)$ with the corresponding value for the mean-field potential $U\left(T, n\left(n_{i d}\right)\right)$ obeying the selfconsistency condition (4), one can improve the total pressure of the mean-field model by matching its repulsive part to the pressure of hard spheres.

The problem, however, arises at low temperatures, while calculating the entropy density for the model with $P_{\text {int }}\left(T, n\left(n_{i d}\right)\right)$ (19). Indeed, for any choice of 
the mean-field potential of the form $U\left(T, n\left(n_{i d}\right)\right)=$ $g(T) f\left(n\left(n_{i d}\right)\right)$ (note that Eq. (19) has such a form) from the thermodynamic identities $s=\frac{\partial p(T, \mu)}{\partial T}$ and $s_{i d}=\frac{\partial p_{i d}(T, \nu)}{\partial T}$, one finds [14]

$s(T, \mu)=\left[s_{i d}+\left[n_{i d} \frac{\partial U}{\partial T}-\frac{\partial P_{\text {int }}}{\partial T}\right]\right]\left[1+b n_{i d}\right]^{-1}=$

$=\left[s_{i d}+\frac{d g(T)}{d T} \int_{0}^{n_{i d}} d \tilde{n} f(n(\tilde{n}))\right]\left[1+b n_{i d}\right]^{-1}$,

where, in deriving Eq. (21) from Eq. (20), we used Eq. (5) to express the interaction pressure $P_{\text {int }}$ in terms of the potential $U\left(T, n\left(n_{i d}\right)\right)=g(T) f\left(n\left(n_{i d}\right)\right)$. Using such an expression, one finds the following derivative:

$\frac{\partial P_{\mathrm{int}}}{\partial T}=n_{i d} f\left(n\left(n_{i d}\right)\right) \frac{d g(T)}{d T}-\frac{d g(T)}{d T} \int_{0}^{n_{i d}} d \tilde{n} f(n(\tilde{n}))$.

Substituting this expression into (20), one gets Eq. (21).

As one can see now from Eq. (21), the mean-field model with the linear $T$ dependence of $U$ or, equivalently, of $P_{\text {int }}$, i.e., $g(T)=T \Rightarrow \frac{d g(T)}{d T}=1$, breaks down the Third Law of thermodynamics. Indeed, at $T=0$, one finds $s_{i d}(T=0, \nu)=0$ by construction, whereas, for the full entropy density, one gets

$s(T=0, \mu)=\left[1+b n_{i d}\right]^{-1} \frac{d g(T)}{d T} \int_{0}^{n_{i d}} d \tilde{n} f(n(\tilde{n})) \neq 0$,

unless $f \equiv 0$. Hence, the mean-field model with the linear $T$ dependence of $P_{\text {int }}$ suggested in [14] may be very good at high temperatures, for which the Boltzmann statistics is valid, but it is unphysical at $T=0$.

Of course, one can repair this defect by choosing a more complicated function $g(T)$, which behaves at high $T$ as $g(T) \sim T$. But its derivative $g^{\prime}(T)$ vanishes at $T=0$, providing the fulfillment of the Third Law of thermodynamics (see an example in Sect. 5 for which $g(T) \sim T^{2}$ at low temperatures). However, in this case, the whole idea to compensate the defects of the VdW EoS by tuning the interacting part of the pressure does not work at low $T$, since, in this case, $P_{\text {int }}=g(T) F\left(n_{i d}\right)$ would vanish faster than the first term staying on the right-hand side of Eq. (17), i.e., the classical part of the pressure $T n_{i d}=T n /(1-b n)$. Thus, we explicitly showed here that, at low $T$, the mean-field models defined by Eqs. (1)-(5) either are unphysical, if $P_{\mathrm{int}}=T F\left(n_{i d}\right)$, or they cannot go beyond the $V d W$ approximation by adjusting their interaction pressure $P_{\text {int }}$.

Such a conclusion can be also applied to the one of two ways to introduce the excluded volume correction into the quantum second virial coefficients discussed in Ref. [34]. Although the model of Ref. [34] contains the scalar mean-fields which modify the masses of particles, the effective potential approach to treat the excluded volume correction of Ref. [34] with the linear $T$ dependence of the repulsive effective potential $W_{i}$ (equivalent to the mean-field potential $-U$ in our notations) of the $i$-th particle sort [see Eqs. (20) and (46) and (47) in [34]] should unavoidably lead to a breakdown of the Third Law of thermodynamics. Therefore, we conclude that such a way to introduce the excluded volume correction into the quantum second virial coefficients discussed in [34] is unphysical. Thus, despite the claims of the author of Ref. [34], such a generalization of the approach [7] to include the hard-core repulsion in quantum systems leads to a problem with the Third Law of thermodynamics. To end this section, we express the traditional virial coefficients $a_{k}^{Q}$ of the quantum VdW gas of Eq. (17) in terms of the classical excluded volume $b$ and the quantum virial coefficients of point-like particles $a_{k}^{(0)}$. Expanding each denominator in Eq. (17) into a series in powers of $n$, one can easily find

$p_{i d}(T, \nu)=T\left[n+\sum_{k=2}^{\infty} a_{k}^{Q} n^{k}\right]$

where

$a_{2}^{Q}=b+a_{2}^{(0)}$,

$a_{3}^{Q}=b^{2}+2 b a_{2}^{(0)}+a_{3}^{(0)}$,

$a_{4}^{Q}=b^{3}+3 b^{2} a_{2}^{(0)}+3 b^{1} a_{3}^{(0)}+a_{4}^{(0)}$,

$a_{k}^{Q}=b^{k-1}+\sum_{l=2}^{k} \frac{(k-1) !}{(l-1) !(k-l) !} b^{k-l} a_{l}^{(0)}$.

If the interaction pressure $P_{\text {int }}\left(T, n_{i d}(n)\right)$ of model (1) can be expanded into the Taylor series of the particle number density $n$ at $n=0$, then one can obtain the 
full quantum virial expansion of this EoS. Note that the coefficients $a_{k}^{(0)}$ for model (1) depend on the temperature only, while specific features of the EoS are stored in $b$ and in $P_{\text {int }}\left(T, n_{i d}(n)\right)$. For example, using the coefficients $b=3.42 \mathrm{fm}^{3}$ and $P_{\text {int }}(T, n)=a_{\text {attr }} n^{2}$ $\left(a_{\mathrm{attr}}=329 \mathrm{MeV} \cdot \mathrm{fm}^{3}\right)$ found in [15] for the quantum VdW EoS of nuclear matter, one can calculate the full quantum second virial coefficient of the model as

$a_{2}^{Q, \text { tot }}=b+a_{2}^{(0)}-\frac{a_{\text {attr }}}{T} \simeq b+\frac{1}{2^{\frac{5}{2}} d_{p}}\left[\frac{2 \pi}{T m_{p}}\right]^{\frac{3}{2}}-\frac{a_{\text {attr }}}{T}$,

where, on the second step of the derivation, we used the non-relativistic expression for the cluster integral $b_{2}^{(0)}(15)$. Taking results from [15], one can find that, for nucleons $\left(d_{p}=4, m_{p}=939 \mathrm{MeV}\right)$, the coefficient $a_{2}^{Q, \text { tot }}(T)$ is zero at $T \simeq 0.32 \mathrm{MeV}$ and $T \simeq 90.5 \mathrm{MeV}$, is negative between these temperatures. Then, above $T \simeq 90.5 \mathrm{MeV}$, it grows almost linearly with $T$ to $a_{2}^{Q, \text { tot }}(T=150 \mathrm{MeV}) \simeq(3.42+0.101-2.19) \mathrm{fm}^{3} \simeq$ $\simeq 1.33 \mathrm{fm}^{3}$ which corresponds to the equivalent hardcore radius $R_{\mathrm{eq}} \simeq 0.46 \mathrm{fm}$ at $T=150 \mathrm{MeV}$. From this estimate, it is evident that the large value of the equivalent hard-core radius $R_{\text {eq }}$ for model [15] is a consequence of the unrealistically large hard-core radius of nucleons $R_{n} \simeq 0.59 \mathrm{fm}$ obtained in [15] (see also a discussion later). In the most advanced version of the hadron resonance gas model, the hard-core radius of nucleons is $0.365 \mathrm{fm}$ [25-27], and, in the IST EoS of the nuclear matter, this radius is below $0.4 \mathrm{fm}$ [35]. It is obvious that a more realistic attraction than the one used in [15] would decrease the values of $R_{\text {eq }}$ and $R_{n}$ to physically more adequate ones. Although the explicit quantum virial expansion (23)-(28) can be used to find the appropriate attraction in order to cure the problems of the VdW EoS and to extend it to higher particle number densities and high/low $T$ values, the true solution of this problem is suggested below.

\section{EoS with Induced Surface Tension}

In order to overcome the difficulties of the quantum VdW EoS at high particle number densities, we suggest the following EoS

$p=p_{i d}\left(T, \nu_{1}\right)-P_{\text {int } 1}\left(T, n_{i d 1}\right)$
$\Sigma=R_{p}\left[p_{i d}\left(T, \nu_{2}\right)-P_{\text {int } 2}\left(T, n_{i d 2}\right)\right]$
$\nu_{1}=\mu-V_{0} p-S_{0} \Sigma+U_{1}\left(T, n_{i d 1}\right)$,

$\nu_{2}=\mu-V_{0} p-\alpha S_{0} \Sigma+U_{2}\left(T, n_{i d 2}\right)$

where $n_{i d A} \equiv \frac{\partial p_{i d}\left(T, \nu_{A}\right)}{\partial \nu_{A}}$ with $A=\{1 ; 2\}, S_{0}=4 \pi R_{p}^{2}$ denotes the proper surface of the hard-core volume $V_{0}$. Equation (29) is an analog of Eq. (1), while the equation for the induced surface tension coefficient $\Sigma$ (30) was first introduced for the Boltzmann statistics in [28]. System (29)-(32) is a quantum generalization of the Boltzmann EoS in the spirit of work [7]. As it was argued above, the temperature-dependent effective potentials considered in [34] may lead to an unphysical behavior at low temperatures. Hence, we would like to study this problem in detail. Below, we will show what is a principal difference of EoS (29)(32) with the second way to include the hard-core repulsion in quantum systems discussed in Ref. [34].

The quantity $\Sigma$ defined by (30) is the surface part of the hard-core repulsion [26]. As it will be shown later, the representation of the hard-core repulsion in pressure (29) in two terms, namely via $-V_{0} p$ and $-S_{0} \Sigma$, instead of a single term $-4 V_{0} p$, as it is done in the quantum VdW EoS, has great advantages and allows one to go beyond the $\mathrm{VdW}$ approximation.

Evidently, the self-consistency conditions for the IST EoS are similar to Eqs. (4) and (5) $(A=\{1 ; 2\})$

$n_{i d A} \frac{\partial U_{A}\left(T, n_{i d A}\right)}{\partial n_{i d A}}=\frac{\partial P_{\operatorname{int~A}}\left(T, n_{i d A}\right)}{\partial n_{i d A}}$.

The model parameter $\alpha>1$ is a switch between the excluded and proper volume regimes. To demonstrate this property, let us consider the quantum distribution function

$$
\begin{aligned}
& \phi_{i d}\left(k, T, \nu_{2}\right) \equiv \frac{1}{e^{\frac{E(k)-\nu_{2}}{T}}+\zeta}= \\
& =\frac{e^{\frac{\nu_{2}-\nu_{1}}{T}}}{e^{\frac{E(k)-\nu_{1}}{T}}+\zeta-\zeta\left[1-e^{\frac{\nu_{2}-\nu_{1}}{T}}\right]}= \\
& =\phi_{i d}\left(k, T, \nu_{1}\right) e^{\frac{\nu_{2}-\nu_{1}}{T}} \times \\
& \times\left\{1+\sum_{l=2}^{\infty}\left[\phi_{i d}\left(k, T, \nu_{1}\right) \zeta\left(1-e^{\frac{\nu_{2}-\nu_{1}}{T}}\right)\right]^{l}\right\},
\end{aligned}
$$

where, in the last step of the derivation, we have expanded the longest denominator above into a series of $\phi_{i d}\left(k, T, \nu_{1}\right) \zeta\left(1-e^{\frac{\nu_{2}-\nu_{1}}{T}}\right)$ powers. Consider two limits of (34), namely $e^{\frac{\nu_{2}-\nu_{1}}{T}} \simeq 1$ and $e^{\frac{\nu_{2}-\nu_{1}}{T}} \rightarrow 0$ for

ISSN 2071-0194. Ukr. J. Phys. 2018. Vol. 63, No. 10 
$\zeta \neq 0$. Then the distribution function $(34)$ can be cast as:

$$
\begin{aligned}
& \phi_{i d}\left(k, T, \nu_{2}\right) \rightarrow \\
& \rightarrow \phi_{i d}\left(k, T, \nu_{1}\right) e^{\frac{\nu_{2}-\nu_{1}}{T}} \begin{cases}\text { for } \zeta \neq 0, & \text { if } e^{\frac{\nu_{2}-\nu_{1}}{T}} \simeq 1, \\
\text { for } \forall \zeta, & \text { if } e^{\frac{\nu_{2}-\nu_{1}}{T}} \rightarrow 0 .\end{cases}
\end{aligned}
$$

Further on, we assume that the inequality

$(\alpha-1) S_{0} \Sigma / n_{i d 2} \gg\left(U_{2}-U_{1}\right) / n_{i d 2}$,

holds in either of the considered limits for $e^{\frac{\nu_{2}-\nu_{1}}{T}}$. Note that, in the case $e^{\frac{\nu_{2}-\nu_{1}}{T}} \simeq 1$, condition (36) is a natural one, because, at low particle densities, it means that the difference of two mean-field potentials $\left(U_{2}-U_{1}\right)$ is weaker than the hard-core repulsion term $(\alpha-1) S_{0} \Sigma$; whereas, for $e^{\frac{\nu_{2}-\nu_{1}}{T}} \rightarrow 0$, it means that such a difference is simply restricted from above for large values of $\Sigma$, i.e., $\max \left\{\left|U_{1}\right| ;\left|U_{2}\right|\right\}<$ $<$ Const $<\infty$. Evidently, in this limit, the mean-field pressures should be also finite, i.e. $\left|P_{\text {int A }}\right|<\infty$.

In the case $e^{\frac{\nu_{2}-\nu_{1}}{T}} \simeq 1$, one immediately recovers the relation

$p_{i d}\left(T, \nu_{2}\right) \simeq e^{\frac{(1-\alpha) S_{0} \Sigma}{T}} p_{i d}\left(T, \nu_{1}\right)$

for $\zeta \neq 0$, which exactly corresponds to the Boltzmann statistics version [26] of system (29)(32). Hence, one recovers the virial expansion of $p_{i d}\left(T, \nu_{1}\right)[26]$ in terms of the particle number density $n_{1}=\left.\frac{\partial p_{i d}\left(T, \nu_{1}\right)}{\partial \mu}\right|_{U_{1}}$, which is calculated under the condition $U_{1}=$ const:

$$
\begin{aligned}
& \frac{p_{i d}\left(T, \nu_{1}\right)}{T n_{1}} \simeq 1+4 V_{0} n_{1}+[16-18(\alpha-1)] V_{0}^{2} n_{1}^{2}+ \\
& +\left[64+\frac{243}{2}(\alpha-1)^{2}-216(\alpha-1)\right] V_{0}^{3} n_{1}^{3}+\ldots
\end{aligned}
$$

Note that, due to the self-consistency condition (33), one finds $\frac{\partial p\left(T, \nu_{1}\right)}{\partial \mu}=\left.\frac{\partial p_{i d}\left(T, \nu_{1}\right)}{\partial \mu}\right|_{U_{1}}$, and, therefore, $n_{1}$ is the physical particle number density.

As it was revealed in [26] for $\alpha=\alpha_{B} \equiv 1.245$, one can reproduce the fourth virial coefficient of the gas of hard spheres exactly, while the value of the third virial coefficient of such a gas is recovered with the relative error about $16 \%$ only. Therefore, for low densities, i.e., for $V_{0} n_{1} \ll 1$, the IST EoS (29)-(32) reproduces the results obtained for $\zeta=0$, if condition (36) is fulfilled.

ISSN 2071-0194. Ukr. J. Phys. 2018. Vol. 63, No. 10
On the other hand, from Eqs. (34) and (35), one sees that, in the limit $e^{\frac{\nu_{2}-\nu_{1}}{T}} \rightarrow 0$, the distribution function $\phi_{i d}\left(k, T, \nu_{2}\right)$ with $\zeta \neq 0$ acquires the Boltzmann form. In this limit, we find $p_{i d}\left(T, \nu_{2}\right) \simeq$ $\simeq p_{i d}\left(T, \nu_{1}\right) e^{\frac{\nu_{2}-\nu_{1}}{T}}$ and $n_{i d 2}^{(0)} \simeq n_{i d 1}^{(0)} e^{\frac{\nu_{2}-\nu_{1}}{T}}$. Using these results and Eq. (36), we can rewrite (30) as

$\Sigma \simeq R_{p}\left[p_{i d}\left(T, \nu_{1}\right) e^{\frac{(1-\alpha) S_{0} \Sigma}{T}}-P_{\operatorname{int} 2}\left(T, n_{i d 2}^{(0)}\right)\right]$.

Here, we use the same notation as in the previous section (see a paragraph before Eq. (14)). From Eq. (38), one can see that, for $\frac{V_{0} p_{i d}\left(T, \nu_{1}\right)}{T} \gg 1$, the surface tension coefficient $\Sigma$ is strongly suppressed compared to $R_{p} p_{i d}\left(T, \nu_{1}\right)$, i.e., one finds

$\Sigma \simeq \frac{T}{S_{0}(\alpha-1)} \ln \left[\frac{R_{p} p_{i d}\left(T, \nu_{1}\right)}{\Sigma}\right] \ll R_{p} p_{i d}\left(T, \nu_{1}\right)$.

Note that, for $\alpha>1$, the condition $e^{\frac{\nu_{2}-\nu_{1}}{T}} \rightarrow 0$ can be provided by $S_{0} \Sigma / T \gg 1$ only. Thus, the second term on the right-hand side of Eq. (38) cannot dominate, since it is finite. It is evident that the inequality $\frac{V_{0} p_{i d}\left(T, \nu_{1}\right)}{T} \gg 1$ also means that $n_{i d 1}^{(0)} V_{0} \gg 1$. Therefore, in this limit, the effective chemical potential (31) can be approximated as

$\nu_{1} \simeq \mu-V_{0} p+U_{1}\left(T, n_{i d 1}^{(0)}\right)$,

i.e., the contribution of the induced surface tension is negligible compared to the pressure. This result means that, for $n_{i d 1}^{(0)} V_{0} \gg 1$, i.e., at high particle densities or for $e^{\frac{\nu_{2}-\nu_{1}}{T}} \rightarrow 0$, the IST EoS corresponds to the proper volume approximation.

On the other hand, Eq. (37) exhibits that, at low densities, i.e., for $e^{\frac{\nu_{2}-\nu_{1}}{T}} \simeq 1$, the IST EoS recovers the virial expansion of the gas of hard spheres up to the fourth power of the particle density $n_{1}$. Therefore, it is natural to expect that, for intermediate values of the parameter $e^{\frac{\nu_{2}-\nu_{1}}{T}} \in[0 ; 1]$, the IST EoS will gradually evolve from the low-density approximation to the high-density one, if condition (36) is obeyed. This is a generalization of the previously obtained result [26] onto the quantum statistics case.

Already from the virial expansion (37), one can see that the case $\alpha=1$ recovers the VdW EoS with the hard-core repulsion. If, in addition, the meanfield potentials are the same, i.e., $U_{2}=U_{1}$ and, consequently, $P_{\text {int } 2}=P_{\text {int } 1}$, then one finds that 
$\nu_{2}=\nu_{1}$ and $\Sigma=R_{p} p\left(T, \nu_{1}\right)$. In this case, the term $V_{0} p+S_{0} \Sigma \equiv 4 V_{0} p$ exactly corresponds to the VdW hard-core repulsion. If, however, $U_{2} \neq U_{1}$, but both mean-field potentials are restricted from above, then the model can deviate from the VdW EoS at low temperatures only, while, at high temperatures, it again corresponds to the VdW EoS. In the case $U_{2}<U_{1}$, this can be easily seen from Eqs. (34) and (35) in the case $e^{\frac{\nu_{2}-\nu_{1}}{T}} \simeq 0$, if one sets $\alpha=1$. Then, using the same logic as in deriving Eq. (38), one can find that $\Sigma \ll R_{p} p_{i d}\left(T, \nu_{1}\right)$. Hence, the effective chemical potential $\nu_{1}$ acquires the form (39). In other words, at low $T$, the surface tension effect becomes negligible, and the IST EoS corresponds to the proper volume approximation, if $e^{\frac{\nu_{2}-\nu_{1}}{T}} \simeq 0$.

Finally, if the inequality $U_{2}>U_{1}$ is valid, then, at low $T$, expansion (34) has to be applied to the distribution function $\phi_{i d}\left(k, T, \nu_{1}\right)$ instead of $\phi_{i d}\left(k, T, \nu_{2}\right)$ and then one arrives at the unrealistic case, since $\Sigma \gg$ $\gg R_{p} p_{i d}\left(T, \nu_{1}\right)$. In this case, the hard-core repulsion would be completely dominated by the induced surface tension term. Hence, even the second virial coefficient would not correspond to the excluded volume of particles.

\section{Going Beyond VdW Approximation}

Let us closely inspect the IST EoS and show explicitly its major differences from the VdW one. For such a purpose in this section, we analyze its effective and true virial expansions and discuss somewhat unusual properties of the entropy density.

\subsection{Effective virial expansion}

First, we analyze the particle densities $n_{1}\left(T, \nu_{1}\right) \equiv$ $\equiv \frac{\partial p\left(T, \nu_{1}\right)}{\partial \mu}$ and $\tilde{n}_{2}\left(T, \nu_{2}\right) \equiv R_{p}^{-1} \frac{\partial \Sigma\left(T, \nu_{2}\right)}{\partial \mu}$. For this purpose, we differentiate Eqs. (29) and (30) with respect to $\mu$ and apply the self-consistency conditions (33)

$n_{1}=n_{i d 1}\left[1-V_{0} n_{1}-S_{0} \frac{\partial \Sigma}{\partial \mu}\right]$,

$\frac{\partial \Sigma}{\partial \mu}=R_{p} n_{i d 2}\left[1-V_{0} n_{1}-\alpha S_{0} \frac{\partial \Sigma}{\partial \mu}\right]$.

Expressing $\frac{\partial \Sigma}{\partial \mu}$ from Eq. (41) and substituting it into (40), one finds the particle number densities $\left(\tilde{n}_{2}\left(T, \nu_{2}\right) \equiv n_{2}\left(1-V_{0} n_{1}\right)\right)$

$n_{1}=\frac{n_{i d 1}\left(1-3 V_{0} n_{2}\right)}{1+V_{0} n_{i d 1}\left(1-3 V_{0} n_{2}\right)}$, $n_{2}=\frac{n_{i d 2}}{1+\alpha 3 V_{0} n_{i d 2}}$

where we used the relation $R_{p} S_{0}=3 V_{0}$ for hard spheres. From Eq. (43) for $n_{2}$, one finds that, for $\alpha>1$, the term $\left(1-3 V_{0} n_{2}\right)$ staying above is always positive, since, taking the limit $n_{i d 2} \rightarrow \infty$ in Eq. (43), one finds the limiting density of $\max \left\{n_{2}\right\}=$ $=\left[3 \alpha V_{0}\right]^{-1}$. Therefore, irrespective of the value of $n_{i d 2} \geq 0$, one finds in the limit $n_{i d 1} V_{0} \gg 1$ that $\max \left\{n_{1}\right\}=V_{0}^{-1}$. This is another way to prove that the limiting density of the IST EoS corresponds to the proper volume limit, since, at high densities, it is four times higher than the one of the VdW EoS. Writing the particle number density $n_{i d 1}$ from Eq. (42) as

$n_{i d 1}=\frac{n_{1}}{\left(1-V_{0} n_{1}\right)\left(1-3 V_{0} n_{2}\right)}$,

one can get the formal virial-like expansion for the IST pressure $p_{i d}\left(T, \nu_{1}\right)(29)$

$\frac{p_{i d}\left(T, \nu_{1}\right)}{T}=\sum_{k=1}^{\infty} \frac{a_{k}^{(0)}}{\left[1-3 V_{0} n_{2}\right]^{k}} \frac{\left[n_{1}\right]^{k}}{\left[1-V_{0} n_{1}\right]^{k}}$,

where the expressions for the coefficients $a_{k}^{(0)}$ are given by Eqs. (9)-(16). This result allows us to formally write the expansion

$\frac{p_{i d}\left(T, \nu_{1}\right)}{T} \equiv \sum_{k=1}^{\infty} a_{k}^{(0), I S T} \frac{\left[n_{1}\right]^{k}}{\left[1-V_{0} n_{1}\right]^{k}}$

with the coefficients $a_{k}^{(0), \text { IST }}=\frac{a_{k}^{(0)}}{\left[1-3 V_{0} n_{2}\right]^{k}}$ which depend not only on $T$, but also on $n_{2}$. Expansions (45) and (46) are the generalizations of the ones used for the EoSs of hard discs [32] and hard spheres [33].

Similarly to deriving Eq. (27), one can get the quantum virial expansion for IST pressure $p_{i d}\left(T, \nu_{1}\right)$ from (46):

$p_{i d}\left(T, \nu_{1}\right)=T \sum_{k=1}^{\infty} a_{k}^{Q, \mathrm{IST}} n_{1}^{k}$,

$a_{k}^{Q, \mathrm{IST}}=\sum_{l=1}^{k} \frac{C_{l}^{(k)}}{\left[1-3 V_{0} n_{2}\right]^{l}}$,

$C_{l}^{(k)}=\frac{(k-1) !}{(l-1) !(k-l) !} V_{0}^{k-l} a_{l}^{(0)}$,

with the coefficients $a_{k}^{Q, \text { IST }}$ which are $T$ - and $n_{2}$-dependent. For the interaction pressure $P_{\text {int } 1}\left(T, n_{i d 1}\right)$

ISSN 2071-0194. Ukr. J. Phys. 2018. Vol. 63, No. 10 
which is expandable in terms of the density $n_{1}$, Eq. (48) can be used to estimate the full quantum virial coefficients of higher orders. Of course, Eq. (47) is not the traditional virial expansion. But the fact that it can be exactly obtained from the grand canonical ensemble formulation of the quantum version of the IST EoS for the third, fourth, and higher order virial coefficients is still remarkable.

\subsection{True quantum virial coefficients}

Now, we consider an example on how to employ results (47)-(49) to estimate the true virial coefficients at low densities and at sufficiently high temperatures which provide the convergence of the virial expansion (47). Apparently, in this case, one can expand the density $n_{2} \simeq B_{1} n_{1}\left(1+B_{2} n_{1}\right)$ in powers of the density $n_{1}$. From our above treatment of the low-density limit $e^{\frac{\nu_{2}-\nu_{1}}{T}} \simeq 1$, it is clear that $B_{1}=1$. Substituting this expansion for $n_{2}$ into Eqs. (47) and (48) and keeping only the terms up to $n_{1}^{2}$, one can get the true quantum virial coefficients $a_{k}^{Q \text {, tot }}$ as

$$
\begin{aligned}
& a_{2}^{Q, \text { tot }}=V_{0}+a_{2}^{(0)}+3 V_{0} B_{1}=4 V_{0}+a_{2}^{(0)}, \\
& a_{3}^{Q, \text { tot }} \simeq 13 V_{0}^{2}+3 V_{0} B_{2}+5 V_{0} a_{2}^{(0)}+a_{3}^{(0)}, \\
& a_{k \geq 3}^{Q, \text { tot }} \simeq \sum_{l=1}^{k} C_{l}^{(k)}+3 V_{0} B_{1} \sum_{l=1}^{k-1} C_{l}^{(k-1)} l+ \\
& +3 V_{0} B_{1} \sum_{l=1}^{k-2} C_{l}^{(k-2)}\left[\frac{3}{2} l(l+1) V_{0} B_{1}+B_{2}\right],
\end{aligned}
$$

and replace the coefficients $a_{k}^{Q, \text { IST }}$ in Eq. (47) with the true quantum virial coefficients $a_{k}^{Q \text {,tot }}$ which depend on $T$ only. Note that the expression for the second virial coefficient $a_{2}^{Q \text {,tot }}$ is exact, while the expressions for the higher order virial coefficients are the approximate ones, which, nevertheless, at high values of temperature are rather accurate. Considering the limit of high temperatures which allows one to ignore the quantum corrections in Eqs. (50) and (51), one can find the coefficients $B_{1}=1$ exactly and $B_{2} \simeq[7-6 \alpha] V_{0}$ approximately by comparing expressions (50) and (51) with the corresponding virial coefficients of the Boltzmann gas in Eq. (37). Substituting the obtained expressions for $B_{1}$ and $B_{2}$ coefficients into Eq. (52), one gets the approximate formula for higher-order virial coefficients $a_{k \geq 3}^{Q \text {,tot }}$ :

$$
\begin{aligned}
& a_{k \geq 3}^{Q, \text { tot }} \simeq \sum_{l=1}^{k} C_{l}^{(k)}+3 V_{0} \sum_{l=1}^{k-1} C_{l}^{(k-1)} l+ \\
& +3 V_{0}^{2} \sum_{l=1}^{k-2} C_{l}^{(k-2)}\left[\frac{3}{2} l(l+1)+(7-6 \alpha)\right]= \\
& =\sum_{l=1}^{k} \frac{(k-1) ! V_{0}^{k-l} a_{l}^{(0)}}{(l-1) !(k-l) !}+3 \sum_{l=1}^{k-1} \frac{(k-2) ! V_{0}^{k-l} a_{l}^{(0)}}{(l-1) !(k-1-l) !} l+ \\
& +3 \sum_{l=1}^{k-2} \frac{(k-3) ! V_{0}^{k-l} a_{l}^{(0)}}{(l-1) !(k-2-l) !}\left[\frac{3}{2} l(l+1)+(7-6 \alpha)\right]
\end{aligned}
$$

where the second equality above is obtained by substituting Eq. (49) for the coefficients $C_{l}^{(k)}$ into the first one.

Comparing now Eq. (53) for the IST EoS and Eq. (27) for the VdW EoS, one can see that the first sum on the right-hand side of (53) is identical to the expression for the VdW quantum virial coefficients with the excluded volume $b=4 V_{0}$ replaced by the proper volume $V_{0}$. Apparently, the other two sums on the right-hand side of (53) are the corrections due to the induced surface tension coefficient.

Note that it is not difficult to get the exact expressions for the third or fourth virial coefficients $a_{k}^{Q, \text { tot }}$ by inserting the higher order terms of the expansion $n_{2}\left(n_{1}\right)$ in powers of the density $n_{1}$ into Eqs. (47) and (48). Although, comparing the coefficients in front of $B_{1}$ and $B_{2}$ in the last sum of Eq. (52), one can see that, even for $l=1$, the coefficient staying before $B_{1}$ is essentially larger than the one staying before $B_{2}$. This means that, at low densities, the role of $B_{2}$ is an auxiliary one, if $\alpha$ is between 1 and 1.5.

\subsection{Virial expansion \\ for compressible spheres}

It is of interest that the $k$-th term

$\frac{1}{\left[1-3 V_{0} n_{2}\right]^{k}} \frac{\left[n_{1}\right]^{k}}{\left[1-V_{0} n_{1}\right]^{k}}$,

staying in sum (45) allows for a non-trivial interpretation. Comparing Eq. (17) and Eq. (45) and recalling the fact that the particle number density $n_{1}$ is proportional to the number of spin-isospin configurations 
$d_{p}$, one can introduce an effective number of such configurations as $d_{p}^{\text {eff }}=\frac{d_{p}}{1-3 V_{0} n_{2}}$ with the simultaneous replacement of $V_{0}$ by the effective proper volume

$V_{0}^{\mathrm{eff}}=V_{0}\left(1-3 V_{0} n_{2}\right)$

in all terms which contain the powers of $\left[1-V_{0} n_{1}\right]$ on the right-hand side of (45). Then, at high densities, the effective number of spin-isospin configurations $d_{p}^{\text {eff }} \leq \frac{\alpha d_{p}}{\alpha-1}$ can be sizably larger than $d_{p}$, while the effective proper volume $V_{0}^{\text {eff }}$ can be essentially smaller than $V_{0}$ (i.e., such effective particles are compressible), if the coefficient $\alpha>1$ is close to 1 . Moreover, one can also establish the equivalent virial expansion of pressure (45) in terms of $\frac{n_{1}}{\left(1-3 V_{0} n_{2}\right)}$ powers. Then, instead of the coefficients $a_{k}^{Q, \text { IST }}$ (48), one would get

$\tilde{a}_{k}^{Q, \mathrm{IST}}=\sum_{l=1}^{k} \frac{(k-1) !}{(l-1) !(k-l) !}\left[V_{0}^{\mathrm{eff}}\right]^{k-l} a_{l}^{(0)}$,

which shows that, at high densities, the contributions of low-order virial coefficients $a_{l}^{(0)}$ into the coefficient $\tilde{a}_{k>1}^{Q, \text { IST }}$ are suppressed due to a decrease of $V_{0}^{\text {eff }}$. Eq. (54) quantifies the source of softness of the IST EoS compared to $\mathrm{VdW}$ one at high densities. It is also interesting that the monotonic decrease of $V_{0}^{\text {eff }}$ at high densities is qualitatively similar to the effect of the Lorentz contraction of a proper volume for relativistic particles [36].

Although the present model does not know anything about the internal structure of considered particles, but the fact that $d_{p}^{\text {eff }}$ increases with the particle number density $n_{2}$ can be an illustration of the in-medium effect that the IST hard-core interaction "produces" the additional (or "enhances" the number of existing) spin-isospin states which are well known in quantum physics as excited states, but with an excitation energy being essentially smaller than the mean value of the particle free energy. In this way, one can see that, at high densities, the IST effectively increases the degeneracy factor of particles. This finding is a good illustration that the claim of Ref. [34] that accounting for the excluded volume correction in the quantum case via the effective degeneracy leads to a reduction of the latter (see a discussion of Eqs. (18) and (19) in [34]) is not a general one. On contrary, a more advanced EoS developed above requires not a reduction of the effective number of degrees of freedom as it is suggested in [34], but their enhancement.

It is apparent that, for $\alpha \gg 1$, the quantities $V_{0}^{\text {eff }}$ and $d_{p}^{\text {eff }}$ are practically independent of $n_{2}$, i.e., in this case, the coefficients $a_{k}^{Q, \text { IST }}$ and $\tilde{a}_{k}^{Q, \text { IST }}$ are the true quantum virial coefficients of the VdW EoS, but with the excluded volume $b=4 V_{0}$ replaced by $V_{0}$.

\subsection{Properties of entropy density}

Next, we study the entropy density of the IST EoS. Similarly to finding the derivatives of Eqs. (29) and (30) with respect to $\mu$, one has to find their derivatives with respect to $T$ in order to get the entropy per particle

$$
\begin{aligned}
& \frac{s_{1}}{n_{1}}=\frac{\left[\frac{\tilde{s}_{i d 1}}{n_{i d 1}}-3 V_{0} n_{2} \frac{\tilde{s}_{i d 2}}{n_{i d 2}}\right]}{\left[1-3 V_{0} n_{2}\right]} \\
& \tilde{s}_{i d A} \equiv s_{i d A}+n_{i d A} \frac{\partial U_{A}}{\partial T}-\frac{\partial P_{\operatorname{int} A}}{\partial T}
\end{aligned}
$$

where the entropy density of point-like particles is defined as $s_{i d A} \equiv \frac{\partial p_{i d}\left(T, \nu_{A}\right)}{\partial T}$ and $A \in\{1 ; 2\}$. If the mean-field potentials of the model have the form

$U_{A}=\sum_{\lambda} g_{A}^{\lambda}(T) f_{A}^{\lambda}\left(n_{i d A}\right)$

and, for $T=0$, their derivatives obey the set of conditions $\frac{d g_{A}^{\lambda}(T)}{d T}=0$, then it is easy to see that the entropy per particle $\frac{s_{1}}{n_{1}}$ also vanishes at $T=0$, i.e. the Third Law of thermodynamics is obeyed under these conditions. In a special case where the interaction mean-field potentials do not explicitly depend on the temperature $T$, the expression for the entropy densities (56) gets simpler, i.e., $\tilde{s}_{i d A}=s_{i d A}$. This case is important for the hadron resonance model and is discussed in the Appendix in some details.

Apparently, to provide a positive value of the entropy per particle $\frac{s_{1}}{n_{1}}$, one has to properly choose the interaction terms in Eqs. (29) and (30). In other words, the Third Law of thermodynamics provides one of the basic constraints to the considered EoS. It is clear that the corresponding necessary conditions should not be very restrictive, because, at low densities, i.e. for $3 V_{0} n_{2} \ll 1$, the coefficient staying in front of the term $\frac{\tilde{s}_{i d 2}}{n_{i d 2}}$ is very small, while, at high densities, it is $\alpha^{-1}<1$ for $\alpha>1$. Although a discussion of such conditions is far beyond the scope of this work, we consider two important cases below.

ISSN 2071-0194. Ukr. J. Phys. 2018. Vol. 63, No. 10 
In the case $U_{2}(T, \rho) \equiv U_{1}(T, \rho)$, condition (36) is valid for any choice of parameters. Then one can show a validity of the inequality $\frac{s_{i d 1}}{n_{i d 1}} \geq \frac{s_{i d 2}}{n_{i d 2}}$, since, for $\alpha>1$, one finds $\nu_{1}>\nu_{2}$. To prove this inequality, one has to take into account that $p_{i d}\left(T, \nu_{A}\right)$, and all its derivatives are monotonically increasing functions of $T$ and $\nu_{A}$. Then, using relations (34) and (35) between the quantum distribution functions, one can show the validity of the inequality $\frac{s_{i d 1}}{n_{i d 1}} \geq \frac{s_{i d} 2}{n_{i d} 2}$ for two limits $e^{\frac{\nu_{2}-\nu_{1}}{T}} \simeq 1$ and $e^{\frac{\nu_{2}-\nu_{1}}{T}} \rightarrow 0$. Similarly, one can introduce an effective parameter of statistics

$\zeta_{\mathrm{eff}} \equiv \zeta-\zeta\left[1-e^{\frac{\nu_{2}-\nu_{1}}{T}}\right]$

and study the quantities for the distribution function $\phi_{i d}\left(k, T, \nu_{2}\right)$ with an effective parameter of statistics $\zeta_{\text {eff }}$. However, one can easily understand that the inequality $\frac{s_{i d 1}}{n_{i d 1}} \geq \frac{s_{i d 2}}{n_{i d 2}}$ cannot be broken down for any value of the exponential $e^{\frac{\nu_{2}-\nu_{1}}{T}}$ obeying the inequalities $0<e^{\frac{\nu_{2}-\nu_{1}}{T}}<1$. This is so, since the pressure of point-like particles and its partial derivatives are monotonic functions of the parameters $T$ and $\nu_{1}$ (or $\nu_{2}$ ), and the non-monotonic behavior of the entropy per particle can be caused by the phase transition, which does not exists for an ideal gas. Note that we do not consider a possible effect of the BoseEinstein condensation. Using the above inequality between the entropies per particle and requiring that $U_{1} \geq 0$ and the inequalities $\frac{d g_{A}^{\lambda}(T)}{d T}>0$ for $T>0$ and $\frac{d g_{A}^{\lambda}(T=0)}{d T}=0$ hold, one can show that $\frac{s_{1}}{n_{1}} \geq \frac{\tilde{s}_{i d 2}}{n_{i d 2}} \geq 0$, using identity (5).

Another important case corresponds to the choice $U_{1}>0$ and $U_{2}<0$ in Eq. (57), i.e., the mean-field $U_{1}$ describes an attraction, while $U_{2}$ represents a repulsion. Clearly, condition (36) in this case is also fulfilled for any choice of parameters. Using the selfconsistency relation (33) or its more convenient form (5), one can find that the term describing the meanfield entropy in $\tilde{s}_{i d 2}$ can be negative, i.e.

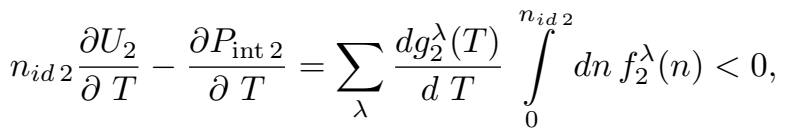

if $g_{2}^{\lambda}(T)>0, \frac{d g_{2}^{\lambda}(T)}{d T}>0$, but $U_{2}<0$ for $T \geq 0$ due to the inequalities $f_{2}^{\lambda}(n)<0$. Such a choice of the interaction allows one to decrease the effective entropy density $\tilde{s}_{i d 2}$ or even to make it negative by tuning the mean-field $U_{2}$ related to the IST coefficient. As a result, this would increase the physical entropy density $s_{1}$. Note that, for the $\mathrm{VdW}$ EoS, this is impossible.

\section{Application to Nuclear and Hadronic Matter}

\subsection{Some important examples}

As a pedagogical example to our discussion, we consider the IST EoS for the nuclear matter and compare it with the VdW EoS (1) having the interaction

$$
\begin{aligned}
& P_{\mathrm{int}}^{\mathrm{VdW}}\left(T, n_{i d}\right)=a\left[\frac{n_{i d}}{1+b n_{i d}}\right]^{2}+T n_{i d}-\frac{g(T) n_{i d}}{1+b n_{i d}}- \\
& -\frac{g(T) b n_{i d}^{2}}{\left[1+b n_{i d}\right]^{2}}-\frac{g(T) B_{3} n_{i d}^{3}}{\left[1+b n_{i d}\right]^{3}}-\frac{g(T) B_{4} n_{i d}^{4}}{\left[1+b n_{i d}\right]^{4}}
\end{aligned}
$$

where the virial coefficients $b, B_{3}$, and $B_{4}$ are introduced above, and the function $g(T) \equiv \frac{T^{2}}{T+T_{\mathrm{SW}}}$ with $T_{\mathrm{SW}}=1 \mathrm{MeV}$ provides the fulfillment of the Third Law of thermodynamics. Note that the term $T n_{i d}$ cancels exactly the first term of the quantum virial expansion for $p_{i d}(T, \nu)$ (see Eq. (17)), while the term $a\left[\frac{n_{i d}}{1+b n_{i d}}\right]^{2}$ in Eq. (59) accounts for an attraction and the other terms proportional to $g(T)$ are the lowest four powers of the virial expansion for the gas of classical hard spheres for $T \gg T_{\mathrm{SW}}$. By construction, such an EoS reproduces, apparently, the four first virial coefficients of the gas of hard spheres at $T \gg T_{\mathrm{SW}}$. Simultaneously, it obeys the Third Law of thermodynamics at $T=0$.

For the IST EoS, we choose $\alpha=1.245$ [26], $P_{\text {int } 1}^{\mathrm{IST}}=$ $=a\left[\frac{n_{i d 1}}{1+b n_{i d 1} 1}\right]^{2}$ and $P_{i n t 2}^{\mathrm{IST}}=0$ with the same constants $a \simeq 329 \mathrm{MeV} \mathrm{fm}{ }^{3}$ and $b=4 V_{0} \simeq 3.42 \mathrm{fm}^{3}$ which were found in [15] for the VdW EoS of nuclear matter $\left(d_{p}=4, m_{p}=939 \mathrm{MeV}\right)$, i.e., we took just the parameters of Ref. [15] for a proper comparison. By construction, the IST EoS and EoS (59) agree very well (within one percent) for $T>120 \mathrm{MeV}$ and particle number densities $n \leq 0.25 \mathrm{fm}^{-3}$. In Fig. 1, we compare three isotherms at $T=19,10$, and $0 \mathrm{MeV}$ of these two EoS. For $T=10 \mathrm{MeV}$, their isotherms agree up to the packing fraction $\eta=V_{0} n \simeq 0.09$ (for the nuclear density $n \leq 0.11 \mathrm{fm}^{-3}$ ), i.e., within the usual range of the VdW EoS applicability [25,26]. However, for $T=0$ and $T=19 \mathrm{MeV}$ isotherms, the both models agree up to the packing fraction $\eta=V_{0} n \simeq 0.03$ only (for $n \leq 0.035 \mathrm{fm}^{-3}$ ), i.e., far below the usual 


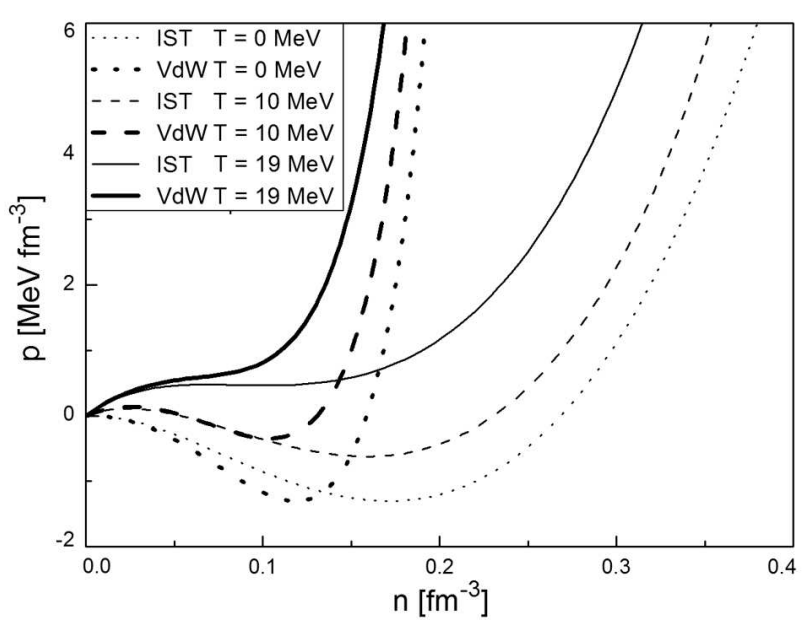

Fig. 1. Behavior of the pressure as a function of the particle number density for isotherms of nuclear matter (see the text for details)

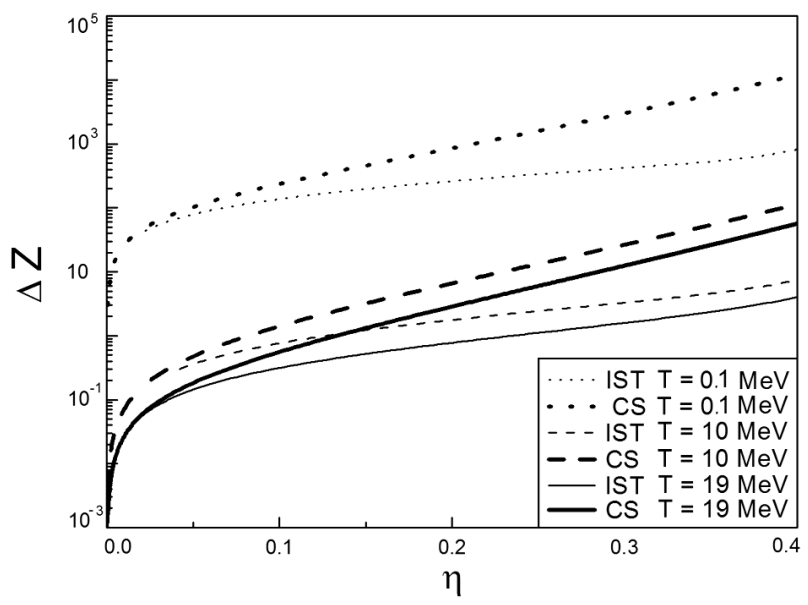

Fig. 2. Packing fraction dependence of the quantum compressibility factors $\Delta Z_{Q}$ of the GVdW EoS and IST EoS (see the text)

range of the VdW EoS applicability due to the important role of the second and higher order quantum virial coefficients $a_{k>2}^{(0)}$ defined by Eqs. (10)-(15). The present example clearly shows that providing the four virial coefficients of the gas of hard spheres for the quantum VdW EoS of Ref. [15] at high temperatures, one can, at most, get a good agreement with the IST EoS for a single value of the temperature, namely for $T=10 \mathrm{MeV}$. Figure 1 also shows that, for the same parameters, the IST EoS is essentially softer that the improved VdW one, hence, it does not require so strong attraction and so strong repulsion to reproduce the properties of normal nuclear matter. This conclusion is supported by the results obtained recently for the nuclear-matter EoS within the IST concept [35].

Recently, an interesting generalization of the quantum VdW EoS (GVdW hereafter) was suggested in [37]. This EoS allows one to go beyond the VdW approximation, but, formally, it is similar to the $\mathrm{VdW}$ models discussed above. In terms of the ideal gas pressure (2), the GVdW pressure can be written as [37] $\left(\eta=V_{0} n\right.$ is the packing fraction):

$p_{\mathrm{G}}(T, \mu)=w(\eta) p_{i d}\left(T, \nu_{\mathrm{G}}\right)-P_{\text {int G }}(n)$,

$\nu_{\mathrm{G}}(\mu, n)=\mu+V_{0} f^{\prime}(\eta) p_{i d}\left(T, \nu_{\mathrm{G}}\right)+U_{\mathrm{G}}(n)$,

where $n$ is the particle density, and the multiplier $w(\eta) \equiv\left(f(\eta)-\eta f^{\prime}(\eta)\right)$ is given in terms of the function $f(\eta)$ which is defined as

$f(\eta)=\left\{\begin{array}{l}f^{\mathrm{VdW}}(\eta)=1-4 \eta, \text { for VdW EoS } \\ f^{\mathrm{CS}}(\eta)=\exp \left[-\frac{(4-3 \eta) \eta}{(1-\eta)^{2}}\right], \text { for CS EoS }\end{array}\right.$

where the function $f^{\mathrm{VdW}}(\eta)$ corresponds to the $\mathrm{VdW}$ case, whereas the function $f^{\mathrm{CS}}(\eta)$ is given for the famous Carnahan-Starling (CS) EoS [17]. The interaction terms of the GVdW EoS are given in terms of a function $u(n): U_{\mathrm{G}}=u(n)+n u^{\prime}(n)$ and $P_{\text {int G }}=$ $=-n^{2} u^{\prime}(n)$. This choice automatically provides the self-consistency condition fulfillment. Since the potentials $U_{\mathrm{G}}$ and $P_{\text {int G }}$ are temperature-independent, the Third Law of thermodynamics is obeyed.

The presence of the function $w(\eta)$ in front of the ideal gas pressure in (60) allows one to reproduce the famous CS EoS [17] at high temperatures, while it creates the problems with formulating the GVdW model for several hard-core radii, since the pressures of point-like particles of kinds 1 and 2 cannot be added to each other, if their functions $w\left(\eta_{1}\right)$ and $w\left(\eta_{2}\right)$ are not the same.

Using the quantum virial expansion (8) and the particle number density expression $n=f(\eta) \times$ $\times n_{i d}\left(T, \nu_{\mathrm{G}}\right)$ [37], for $P_{\mathrm{IG}} \equiv w(\eta) p_{i d}\left(T, \nu_{\mathrm{G}}\right)$, one ob-

$P_{\mathrm{IG}}=w(\eta) T\left[\frac{n}{f(\eta)}+\sum_{l=2}^{\infty} a_{l}^{(0)}\left[\frac{n}{f(\eta)}\right]^{l}\right]$
$\frac{w(\eta)}{f(\eta)}=\left\{\begin{array}{l}\frac{1}{1-4 \eta} \equiv \frac{1}{f^{\mathrm{VdW}}(\eta)}, \text { for } \mathrm{VdW} \mathrm{EoS}, \\ \frac{1+\eta+\eta^{2}-\eta^{3}}{(1-\eta)^{3}}, \text { for CS EoS. }\end{array}\right.$

ISSN 2071-0194. Ukr. J. Phys. 2018. Vol. 63, No. 10 
Although this effective expansion can be used to derive the true virial expansion for the CS parametrization of the GVdW EoS (for the VdW one, it is given above), the result is cumbersome. Nevertheless, these equations show that, due to the multiplier $w(\eta)$, the first term of the quantum virial expansion in Eqs. (63), (8), (17), and (47), i.e., the classical term, exactly reproduces the pressure by the corresponding classical EoS. Hence, all other terms in Eqs. (8), (17), (47), and (63) are the quantum ones. A direct comparison of the IST with $\alpha=1.245$ and CS EoS for classical gases shows that, for packing fractions $\eta>0.22$, the IST EoS is softer than the CS one $[25,26]$. Figure 2 depicts the quantum compressibility factors

$\Delta Z_{Q}^{\mathrm{CS}}(\eta)=\frac{P_{\mathrm{IG}}-w(\eta) T n_{i d}\left(T, \nu_{\mathrm{G}}\right)}{T n}$

for the CS EoS of the GVdW model and the one for the IST EoS defined similarly

$\Delta Z_{Q}^{\mathrm{IST}}(\eta)=\frac{p_{i d 1}-T n_{i d 1}\left(T, \nu_{1}\right)}{T n_{1}}$

taken both for the same parameters $b=3.42 \mathrm{fm}^{3}$, $P_{\text {int G }}(T, n)=a_{\text {attr }} n^{2}$ with $a_{\text {attr }}=329 \mathrm{MeV} \cdot \mathrm{fm}^{3}$ (see [37] for more details). As one can see from Fig. 2, the quantum compressibility factors of these EoS differ essentially for $\eta \geq 0.05$. Therefore, for $\eta \geq 0.1$, both the classical and quantum parts of the IST pressure with $\alpha=1.245$ [26] are essentially softer than the corresponding terms of the CS version of the GVdW model of Ref. [37]. One can easily understand such a conclusion comparing expansions (63) and (45). Since, for the same packing fraction $\eta \geq 0.1$, the function $f^{\mathrm{CS}}(\eta)$ of the CS version of the GVdW EoS vanishes essentially faster than the term $\left[1-3 V_{0} n_{2}\right]\left[1-V_{0} n_{1}\right]$ of the IST EoS, each term proportional to $n^{k}$ in (63) with $k>1$ is larger than the corresponding term proportional to $n_{1}^{k}=n^{k}$ in (45). It is necessary to note that such a property is very important, because the softer EoS provides a wider range of thermodynamic parameters for which the EoS is causal, i.e. its speed of sound is smaller than the speed of light.

\subsection{Constraints on nuclear matter properties}

It is appropriate to discuss the most important constraints on the considered mean-field models which

ISSN 2071-0194. Ukr. J. Phys. 2018. Vol. 63, No. 10 are necessary to describe the strongly interacting matter properties. According to Eqs. (17), (47), and (63), the fermionic pressure for the considered EoS consists of three contributions: the classical pressure (the first term on the right-hand side of (17), (47), and $(63))$, the quantum part of the pressure and the mean-field $P_{\text {int }}$. At temperatures below $1 \mathrm{MeV}$, the classical part is negligible, but the usage of virial expansions discussed above is troublesome due to the convergency problem. Since the exact parametrization of the function $P_{\text {int }}$ on the particle number density of nucleons is not known, it is evident that all considered models are effective by construction. To fix their parameters, one has to reproduce the usual properties of normal nuclear matter, i.e. to get a zero value for the total pressure at the normal nuclear density $n_{0} \simeq 0.16 \mathrm{fm}^{-3}$ and the binding energy $W=-16 \mathrm{MeV}$ at this density [1]. Similarly to the high-temperature case discussed at the end of Section 2, there exists a freedom of parametrizing the hard-core radius of nucleons, since the attraction pressure can be always adjusted to reproduce the properties of normal nuclear matter and, therefore, all the model parameters are also effective by construction.

However, in addition to the properties of normal nuclear matter, there is the so-called flow constraint at nuclear densities $n=(2-5) n_{0}$ [38], which sets strong restrictions on the model pressure dependence on the nuclear particle density and requires a rather soft EoS at these densities. Hence, it can be used to determine the parameters of a realistic EoS at high nuclear densities and $T=0$. Traditionally, such a constraint creates troubles for the relativistic meanfield EoS based on the Walecka model [4,39, 40].

The validity of this statement can be seen from Ref. [39] in which it is shown that only 104 of such EoSs out of 263 analyzed in [39] are able to obey the flow constraint despite the fact that they have 10 or even more adjustable parameters. At the same time, as one can see from the simplest realization of the IST EoS suggested in Ref. [35], the 4-parameter EoS is able to simultaneously reproduce all properties of normal nuclear matter and the flow constraint. Furthermore, the IST EoS is able not only to reproduce the flow constraint, but, simultaneously, it is able to successfully describe the neutron star properties with the masses more than two Solar ones [41], which sets another strong constraint on the stiffness 
of the realistic EoS at high particle densities and the zero temperature. On the other hand, Fig. 2 shows that the existing CS version of the GVdW EoS of Ref. [37] is very stiff, and, hence, it will also have troubles to obey the flow constraint [38].

\subsection{Constraints on hadronic matter properties}

From the virial expansions of all models discussed here, one sees that the EoS calibration on the properties of nuclear matter at low $T$ and at high densities involves mainly the quantum and mean-field pressures. But, unfortunately, it also fixes the parameters of the classical pressure at higher temperatures. It is, however, clear that the one-component mean-field models of nuclear matter cannot be applied at temperatures above $50 \mathrm{MeV}$, since one has to include the mesons, other baryons, and their resonances [31, 42].

Moreover, at high temperatures, the mean-fields and the parameters of interaction should be re-calibrated because the very fact of resonance existence already corresponds to a partial account for the interaction [42]. For many years, it is well known that, for temperatures below $170 \mathrm{MeV}$ and densities below $n_{0}$, the mixture of stable hadrons and their resonances whose interaction is taken into account by the quantum second virial coefficients behaves as a mixture of nearly ideal gases of stable particles. The latter, in this case, includes both the hadrons and the resonances, but taken with their averaged masses [42]. The main reason for such a behavior is rooted in a nearly complete cancellation between the attraction and repulsion contributions. The resulting deviation from the ideal gas (a weak repulsion) is usually described in the hadron resonance gas model (HRGM) [19-27] by the classical second virial coefficients.

Nevertheless, such a repulsion is of principal importance for the HRGM. Otherwise, if one considers a mixture of ideal gases of all known hadrons and their resonances, then, at high temperatures, the pressure of such a system will exceed the one of the ideal gas of massless quarks and gluons [43]. Since such a behavior contradicts the lattice version of quantum chromodynamics, the (weak) hard-core repulsion in the HRGM is absolutely necessary. Moreover, to our best knowledge, there is no other approach which is able to include all known hadronic states into consideration and to be consistent with the thermodynamics of lat- tice quantum chromodynamics at low-energy densities and which, simultaneously, would not contradict it at the higher ones.

Therefore, it seems that the necessity of a weak repulsion between the hadrons is naturally encoded in the smaller values of their hard-core radii $\left(R_{p}<\right.$ $<0.4 \mathrm{fm}$ ) obtained within the HRGM compared to the larger hard-core radius of nucleons in nuclear matter $R_{n} \geq 0.52 \mathrm{fm}$ found in [37]. This conclusion is well supported by the recent simulations of the neutron star properties with masses more than two Solar ones [41] which also favors the nucleon hard-core radii below than $0.52 \mathrm{fm}$. Furthermore, the small values of the hard-core radii provide the fulfillment of the causality condition in the hadronic phase $[25,26,41,46]$, while a possible break of causality occurs in the region, where the hadronic degrees of freedom are not relevant [46]. Hence, in contrast to Ref. [37], we do not see any reason to believe that the mean-field model describing the nuclear matter properties may set any strict conditions on the hadronic hard-core radii of the HRGM.

Moreover, we would like to point out that a great success achieved recently by the HRGM [19-27] sets a strong restriction on any model of hadronic phase which is claimed to be realistic. The point is that, at the chemical freeze-out curve $\mu=\mu_{\mathrm{CFO}}(T)$, the mean-field interaction term of pressure (1) or (29) must vanish. Otherwise, one would need a special procedure to transform the mean-field potential energy into the masses and kinetic energy of non-interacting hadrons (the kinetic freeze-out problem $[44,45]$ ). The existing versions of the HRGM do not face such a problem, since this model has the hard-core repulsion only, while the mean-field interaction in it is set to zero [19-27]. Due to such a choice of the interaction, the HRGM has the same energy per particle as an ideal gas. Hence, it can be tuned to describe the existing experimental hadronic multiplicities in central nuclear collisions from the lower AGS collision energy $\sqrt{s_{N N}}=2.76 \mathrm{GeV}$ to the ALICE center of mass energy $\sqrt{s_{N N}}=2.76 \mathrm{TeV}$ with the total quality of fit $\chi^{2} /$ dof $\simeq 1.04[25,26]$.

Therefore, any realistic hadronic EoS of hadronic matter should be able to reproduce the pressure, entropy, and all charge densities obtained by the HRGM at the chemical freeze-out curve $\mu=\mu_{\mathrm{CFO}}(T)$. In particular, for the mean-field models discussed here, it means that they should be extended in order to

ISSN 2071-0194. Ukr. J. Phys. 2018. Vol. 63, No. 10 
include all other hadrons and, at the curve $\mu=$ $=\mu_{\mathrm{CFO}}(T)$, the total interaction pressure must vanish, i.e., $P_{\text {int }}=0$, since it does not exist in the HRGM.

In other words, if, at the chemical freeze-out curve, such a model EoS has a non-vanishing attraction, then it must have an additional repulsion to provide $P_{\text {int }}=0$. Only this condition will help one to avoid a hard mathematical problem of kinetic freeze-out to convert the interacting particles into a gas of free streaming particles $[44,45]$, since the HRGM with the hard-core repulsion and with vanishing mean-field interaction has the same energy per particle as an ideal gas. Due to its importance, we analyzed the IST EoS in Appendix and show that this EoS also possesses such a property. The condition $P_{\text {int }}=0$ at the chemical freeze-out curve will make a direct connection between the realistic EoS and the hadron multiplicities measured in heavy ion collisions. It is clear that, without $T$-dependent mean-field interaction $P_{\text {int }}$, such a condition cannot be fulfilled.

Despite many valuable results obtained with the HRGM, the hard-core radii are presently well established for the most abundant hadrons only, namely, for pions $\left(R_{\pi} \simeq 0.15 \mathrm{fm}\right)$, the lightest $\mathrm{K}^{ \pm}$-mesons $\left(R_{K} \simeq 0.395 \mathrm{fm}\right)$, nucleons $\left(R_{p} \simeq 0.365 \mathrm{fm}\right)$, and the lightest (anti) $\Lambda$-hyperons $\left(R_{\Lambda} \simeq 0.085 \mathrm{fm}\right)$ $[25,26]$. Nevertheless, we hope for that the new highquality data on the yields of many strange hadrons recently measured by the ALICE Collaboration at CERN [47] at the center of mass energy $\sqrt{s_{N N}}=$ $=2.76 \mathrm{TeV}$ and the ones which are expected to be measured during the Beam Energy Scan II at RHIC BNL (Brookhaven) [48], and at the accelerators of new generations, i.e., at NICA JINR (Dubna) [49,50] and FAIR GSI (Darmstadt) [51,52] will help us to determine their hard-core radii with high accuracy. We have to add only that the IST EoS for quantum gases is well suited for such a task due to the additive pressure $p_{i d}\left(T, \nu_{1,2}\right)$, whereas the generalization of the CS EoS of Ref. [37] to a multicomponent case looks rather problematic, since the CS EoS [17] is the onecomponent EoS by construction.

\section{Conclusions}

The self-consistent generalization of the IST EoS for quantum gases is worked out. It is shown that, with this EoS, one can go beyond the $\mathrm{VdW}$ approximation at any temperature. The restrictions on the tempera- ture dependence of the mean-field potentials are discussed. It is found that, at low temperatures, these potentials either should be $T$-independent or should vanish faster than the first power of the temperature providing the fulfillment of the Third Law of thermodynamics. The same is true for the quantum VdW EoS. Hence, the idea to improve the quantum VdW EoS by tuning the interaction part of the pressure $[14,15]$ is disproved for low temperatures $T$ : if this part of the pressure is linear in $T$, then the VdW EoS breaks down the Third Law of thermodynamics; if it vanishes faster than the first power of $T$, then the interaction part of the pressure is useless, since it vanishes faster than the first term of the quantum virial expansion. An alternative EoS [37] allowing one to abandon the $\mathrm{VdW}$ approximation for nuclear matter is analyzed here, and it is shown that, for the same parameters, the IST EoS is softer at low temperatures at packing fractions $\eta \geq 0.05$.

The virial expansions for the quantum $\mathrm{VdW}$ and IST EoS are established, and the explicit expressions for all quantum virial coefficients, exact for $\mathrm{VdW}$ and approximative ones for the IST EoS, are given. Therefore, for the first time, the analytical expressions for the third and fourth quantum virial coefficients are derived for the EoS which is more realistic than the VdW one. The source of softness of the IST EoS is demonstrated, by using the effective virial expansion for the effective proper volume which turns out to be compressible. The generalization of the traditional virial expansions for the mixtures of particles with different hard-core radii is straightforward.

The general constraints on the realistic EoS for nuclear and hadronic matter are discussed. We hope for that, by using the revealed properties of the IST EoS for quantum gases, it will be possible to go far beyond the traditional VdW approximation, and, due to its advantages, this EoS will become a useful tool for heavy ion physics and for nuclear astrophysics. Furthermore, we hope for that the developed EoS will help us to determine the hard-core radii of hadrons from the new high-quality ALICE data and the ones which will be measured at RHIC, NICA, and FAIR.

The authors appreciate the valuable comments of D.B. Blaschke, R. Emaus, B.E. Grinyuk, D.R. Oliinychenko, and D.H. Rischke. K.A.B., A.I.I., V.V.S. and G.M.Z. acknowledge a partial support of the 
National Academy of Sciences of Ukraine (project No. 0118U003197). V.V.S. thanks the Fundação para a Ciência e Tecnologia (FCT), Portugal, the Multidisciplinary Center for Astrophysics (CENTRA), Instituto Superior Técnico, Universidade de Lisboa, for the partial financial support through the Grant No. UID/FIS/00099/2013. The work of A.I. was performed within the project SA083P17 of Universidad de Salamanca launched by the Regional Government of Castilla y Leon and the European Regional Development Fund.

\section{APPENDIX}

Here, we consider a special choice of the mean-field potentials which are temperature-independent, i.e., $U_{A}=U_{A}\left(n_{i d A}\right)$ and show that, at low particle densities, the energy per particle of such an EoS coincides with the one of the ideal gas. The analysis is made for a single sort of particles, but it is evident that a generalization to the multicomponent case is straightforward.

For the considered choice of the mean-field potentials, Eq. (55) for the entropy per particle becomes

$\frac{s_{1}}{n_{1}}=\frac{\left[\frac{s_{i d 1}}{n_{i d 1}}-3 V_{0} n_{2} \frac{s_{i d 2}}{n_{i d 2}}\right]}{\left[1-3 V_{0} n_{2}\right]} \simeq \frac{s_{i d 1}}{n_{i d 1}}$,

where, in the first step, we applied the relation $\tilde{s}_{i d A}=s_{i d A}$ with $A \in\{1 ; 2\}$ to Eq. (55), while, in the second step, we used an approximation $\frac{s_{i d 2}}{n_{i d 2}} \simeq \frac{s_{i d 1} 1}{n_{i d 1}}$. The latter result follows from condition (36). Then, in the low-density limit, i.e., for $e^{\frac{\nu_{2}-\nu_{1}}{T}} \simeq 1$, one gets relation (35) for the distribution functions $\phi_{i d}\left(k, T, \nu_{2}\right)$ and $\phi_{i d}\left(k, T, \nu_{1}\right)$ which can be approximated further on as $\phi_{i d}\left(k, T, \nu_{2}\right) \simeq \phi_{i d}\left(k, T, \nu_{1}\right)$. Therefore, one finds $p_{i d}\left(T, \nu_{2}\right) \simeq p_{i d}\left(T, \nu_{1}\right), n_{i d}\left(T, \nu_{2}\right) \simeq n_{i d}\left(T, \nu_{1}\right)$ and $s_{i d}\left(T, \nu_{2}\right) \simeq s_{i d}\left(T, \nu_{1}\right)$.

The energy per particle for EoS (29) can be found from the thermodynamic identity

$\frac{\epsilon_{1}}{n_{1}}=T \frac{s_{1}}{n_{1}}+\mu-\frac{p(T, \mu)}{n_{1}}$.

Expressing the chemical potential $\mu$ via an effective one $\nu_{1}$ from Eq. (31), one can write $\mu=\nu_{1}+V_{0} p_{i d 1}-V_{0} P_{\text {int } 1}+3 V_{0} p_{i d 2}-$ $-3 V_{0} P_{\text {int } 2}-U_{1}$. Substituting this result into Eq. (66), one finds

$\frac{\epsilon_{1}}{n_{1}} \simeq T \frac{s_{i d 1}}{n_{i d 1}}+\nu_{1}-U_{1}+\left[V_{0}-\frac{1}{n_{1}}\right]\left(p_{i d 1}-P_{\text {int } 1}\right)+$

$+3 V_{0}\left(p_{i d 2}-P_{\text {int } 2}\right)$

where Eq. (65) was also used. Approximating the particle number density $n_{1}$ in Eq. (42) as

$n_{1} \simeq \frac{n_{i d 1}}{1+V_{0} n_{i d 1}+3 V_{0} n_{2}}$,

and substituting it into Eq. (67), one obtains

$\frac{\epsilon_{1}}{n_{1}} \simeq \frac{\epsilon_{i d 1}}{n_{i d 1}}+3 V_{0} n_{2}\left[\frac{p_{i d 2}}{n_{2}}-\frac{p_{i d 1}}{n_{i d 1}}\right]-U_{1}-$

\section{8}

$-\left[V_{0}-\frac{1}{n_{1}}\right] P_{\text {int } 1}-3 V_{0} P_{\text {int } 2}$,

where we applied the thermodynamic identity (66) to the energy per particle for a gas of point-like particles with the chemical potential $\nu_{1}$. To simplify the evaluation, we assume for the moment that all mean-field interaction terms obey the equality

$\frac{\left(1-V_{0} n_{1}\right)}{n_{1}} P_{\text {int } 1}\left(n_{i d 1}\right)-3 V_{0} P_{\text {int } 2}\left(n_{i d 2}\right)=U_{1}\left(n_{i d 1}\right)$.

Using the first two terms of the virial expansion (8) in Eq. (69) for the pressures $p_{i d 1}$ and $p_{i d 2}$ and Eq. (43) for $n_{2}$, one finds

$\frac{p_{i d 2}}{n_{2}}-\frac{p_{i d 1}}{n_{i d 1}} \simeq T\left[\left(1+a_{2}^{(0)} n_{i d 2}\right)\left(1+3 \alpha V_{0} n_{i d 2}\right)-\right.$

$\left.-\left(1+a_{2}^{(0)} n_{i d 1}\right)\right] \simeq T\left(1+a_{2}^{(0)} n_{i d 1}\right) 3 \alpha V_{0} n_{i d 1}$,

where, in the last step of the derivation, we used the lowdensity approximation $n_{i d 2} \simeq n_{i d 1}$. Finally, under condition (70), Eq. (69) acquires the form

$\frac{\epsilon_{1}}{n_{1}} \simeq \frac{\epsilon_{i d 1}}{n_{i d 1}}+9 \alpha V_{0}^{2} n_{2} n_{i d 1} T\left(1+a_{2}^{(0)} n_{i d 1}\right)$.

Since the typical packing fractions $\eta=V_{0} n_{1} \simeq V_{0} n_{2} \simeq V_{0} n_{i d 1}$ of the hadron resonance gas model at the chemical freeze-out do not exceed the value 0.05 [25], the second term on the righthand side of Eq. (72) is not larger than

$0.025 \alpha T\left(1+a_{2}^{(0)} n_{i d 1}\right)$.

Comparing this estimate with the energy per particle for the lightest hadrons, i.e., for pions, in the non-relativistic limit $\left.\frac{\epsilon_{\text {id } 1}}{n_{\text {id } 1}}\right|_{\pi} \simeq m_{\pi}+\frac{3}{2} T$ (here, $m_{\pi} \simeq 140 \mathrm{MeV}$ ), one can be sure that, for temperatures at which the hadron gas exists, i.e., for $T<160 \mathrm{MeV}$, term (73) is negligible. Hence, one finds $\frac{\epsilon_{1}}{n_{1}} \simeq \frac{\epsilon_{i d 1}}{n_{i d 1}}$ with high accuracy.

Now, we discuss condition (70). It is apparent that, in the general case, it can hold, if the mean-field interaction is absent, i.e., $U_{1}=U_{2}=0$ and $P_{\text {int } 1}=P_{\text {int } 2}=0$. This is exactly the case of the hadron resonance gas model. However, one might think that there exists a special case for which Eq. (70) is the simple differential equation for two independent variables $n_{i d} 1$ and $n_{i d 2}$. Let us show that this is impossible. First, with the help of Eq. (42), we rewrite the term $\frac{\left(1-V_{0} n_{1}\right)}{n_{1}}=\left[n_{i d 1}(1-\right.$ $\left.\left.-3 V_{0} n_{2}\right)\right]^{-1}$. Then Eq. (70) can be cast as

$\frac{P_{\text {int } 1}\left(n_{i d 1}\right) / n_{i d 1}}{\left(1-3 V_{0} n_{2}\left(n_{i d 2}\right)\right)}-3 V_{0} P_{\text {int } 2}\left(n_{i d 2}\right)=U_{1}\left(n_{i d 1}\right)$.

From this equation, one sees that the only possibility to decouple the dependences on $n_{i d 1}$ and $n_{2}$ in the first term above is to assume that $P_{\operatorname{int} 1}=C n_{i d 1}$ where $C$ is some constant. However, in this case, one finds that the $n_{i d 1^{-}}$ dependence of the right-hand side of Eq. (74) remains, since $U_{1}=C \ln \left(n_{i d 1}\right)$. Therefore, there is a single possibility to decouple the functional dependence of $n_{i d 1}$ from $n_{2}$, namely, $C=0$ which means that $P_{\text {int } 2}=0$.

One can, however, consider Eq. (74) under the low-density approximation, by assuming that $n_{i d 2}=n_{i d 1}$. In this case, Eq. (74) defines the functional dependence of $P_{\operatorname{int} 2}\left(n_{i d 1}\right)$ for

ISSN 2071-0194. Ukr. J. Phys. 2018. Vol. 63, No. 10 
any reasonable choice of the potential $U_{1}\left(n_{i d 1}\right)$. Note that, in this case, the function $P_{\text {int } 2}\left(n_{i d 1}\right)$ can be rather complicated even for the simplest choice of $U_{1}\left(n_{i d 1}\right)$. Hence, the practical realization of dependence (74) seems to be problematic. Therefore, the most direct way to avoid the problem to convert the interacting particles into the free streaming ones $[44,45]$ is to use only the hard-core repulsion between hadrons and set all other interactions at the chemical freeze-out to zero.

1. N.K. Glendenning. Compact Stars (Springer, 2000).

2. S. Typel, G. Röpke, T. Klähn, D. Blaschke, H.H. Wolter. Composition and thermodynamics of nuclear matter with light clusters. Phys. Rev. C 81, 015803 (2010) and references therein.

3. S. Benic, D. Blaschke, D.E. Alvarez-Castillo, T. Fischer, S. Typel. A new quark-hadron hybrid equation of state for astrophysics I. High-mass twin compact stars. Astron. Astrophys. 577, A40 (2015).

4. J.D. Walecka. A theory of highly condensed matter. Annals Phys. 83, 491 (1974).

5. J. Zimanyi et al. An interpretable family of equations of state for dense hadronic matter. Nucl. Phys. A 484, 647 (1988).

6. K.A. Bugaev, M.I. Gorenstein. Thermodynamically selfconsistent class of nuclear matter EOS and compression shocks in relativistic nuclear collisions. Z. Phys. C 43, 261 (1989).

7. D.H. Rischke, M.I. Gorenstein, H. Stöcker, W. Greiner. Excluded volume effect for the nuclear matter equation of state. Z. Phys. C 51, 485 (1991).

8. T.H.R. Skyrme. CVII. The nuclear surface. Phil. Mag. 1, 1043 (1956).

9. T.H.R. Skyrme. The effective nuclear potential. Nucl. Phys. 9, 615 (1959).

10. J.P. Hansen, I.R. McDonald. Theory of Simple Liquids (Academic, 2006).

11. L. Van Hove. Quelques proprietes generales de l'integrale de configuration D'un systeme de particules avec interaction. Physica 15, 951 (1949).

12. L. Van Hove. Sur l'integrale de configuration pour les systemes de particules a une dimension. Physica 16, 137 (1950).

13. M.I. Gorenstein, D.H. Rischke, H. Stöcker, W. Greiner, K.A. Bugaev. A self-consistent equation of state for nuclear matter. J. Phys. G 19, L69 (1993).

14. D. Anchishkin, V. Vovchenko. Mean-field approach in the multi-component gas of interacting particles applied to relativistic heavy-ion collisions. arXiv:1411.1444 [nucl-th] and references therein.

15. V. Vovchenko, D.V. Anchishkin, M.I. Gorenstein. Van der Waals equation of state with Fermi statistics for nuclear matter. Phys. Rev. C 91, 064314 (2015).

16. K. Redlich, K. Zalewski. Thermodynamics of van der Waals fluids with quantum statistics. Acta Phys. Polon. $B$ 47, 1943 (2016).

ISSN 2071-0194. Ukr. J. Phys. 2018. Vol. 63, No. 10
17. N.F. Carnahan, K.E. Starling. Equation of state for nonattracting rigid spheres. J. Chem. Phys. 51, 635 (1969).

18. K. Huang. Statistical Mechanics (Wiley, 1963).

19. A. Andronic, P. Braun-Munzinger, J. Stachel. Hadron production in central nucleus-nucleus collisions at chemical freeze-out. Nucl. Phys. A 772, 167 (2006) and references therein.

20. K.A. Bugaev, D.R. Oliinychenko, A.S. Sorin, G.M. Zinovjev. Simple solution to the strangeness horn description puzzle. Eur. Phys. J. A 49, 30 (2013).

21. D.R. Oliinychenko, K.A. Bugaev, A.S. Sorin. Investigation of hadron multiplicities and hadron yield ratios in heavy ion collisions. Ukr. J. Phys. 58, 211 (2013).

22. K.A. Bugaev et al. Chemical freeze-out of strange particles and possible root of strangeness suppression. Europhys. Lett. 104, 22002 (2013).

23. J. Stachel, A. Andronic, P. Braun-Munzinger, K. Redlich. Confronting LHC data with the statistical hadronization model. J. Phys. Conf. Ser. 509, 012019 (2014) and references therein.

24. V.V. Sagun. $\Lambda$-anomaly in the hadron chemical freeze-out. Ukr. J. Phys. 59, 755 (2014).

25. K.A. Bugaev et al. Going beyond the second virial coefficient in the hadron resonance gas mode. Nucl. Phys. A 970, 133 (2018).

26. V.V. Sagun et al. Hadron resonance gas model with induced surface tension. Eur. Phys. J. A 54, 100 (2018).

27. K.A. Bugaev et al. Threshold collision energy of the QCD phase diagram tricritical endpoint. Phys. Part. Nucl. Lett. 15, 210 (2018).

28. V.V. Sagun, K.A. Bugaev, A.I. Ivanytskyi, I.N. Mishustin. The statistical multifragmentation model for liquid-gas phase transition with a compressible nuclear liquid. Nucl. Phys. A 924, 24 (2014).

29. K.A. Bugaev, M.I. Gorenstein, I.N. Mishustin, W. Greiner. Exactly soluble model for nuclear liquid-gas phase transition. Phys. Rev. C 62, 044320 (2000).

30. J.P. Bondorf et al. Statistical multifragmentation of nuclei. Phys. Rep. 257, 131 (1995).

31. A. Kostyuk, M.I. Gorenstein, H. Stöcker, W. Greiner. Second cluster integral and excluded volume effects for the pion gas. Phys. Rev. C 63, 044901 (2001).

32. J. Kolafa, M. Rottner. Simulation-based equation of state of the hard disk fluid and prediction of higher-order virial coefficients. Mol. Phys. 104, 3435 (2006).

33. B. Barboy, W.M. Gelbart. Series representation of the equation of state for hard particle fluids. J. Chem. Phys. 71, 3053 (1979).

34. S. Typel. Variations on the excluded-volume mechanism. Eur. Phys. J. A 52, 16 (2016).

35. A.I. Ivanytskyi, K.A. Bugaev, V.V. Sagun, L.V. Bravina, E.E. Zabrodin. Influence of flow constraint on the properties of nuclear matter critical endpoint. Phys. Rev. C 97, 064905 (2018).

36. K.A. Bugaev. The Van-der-Waals gas EOS for the Lorentz contracted spheres. Nucl. Phys. A 807, 251 (2008) and references therein. 
37. V. Vovchenko. Equations of state for real gases on the nuclear scale. Phys. Rev. C 96, 015206 (2017).

38. P. Danielewicz, R. Lacey, W.G. Lynch. Determination of the equation of state of dense matter. Science 298, 1592 (2002).

39. M. Dutra et al. Relativistic mean-field hadronic models under nuclear matter constraints. Phys. Rev. C 90, 055203 (2014) and references therein.

40. O. Lourenco, M. Dutra, D.P. Menezes. Critical parameters of consistent relativistic mean-field models. Phys. Rev. C 95, 065212 (2017).

41. V.V. Sagun, I. Lopes. Neutron stars: A novel equation of state with induced surface tension. Astrophys. J 850, 75 (2017).

42. R. Venugopalan, M. Prakash. Thermal properties of interacting hadrons. Nucl. Phys. A 546, 718 (1992).

43. L.M. Satarov, M.N. Dmitriev, I.N. Mishustin. Equation of state of hadron resonance gas and the phase diagram of strongly interacting matter. Phys. Atom. Nucl. 72, 1390 (2009).

44. K.A. Bugaev. Shock-like freeze-out in relativistic hydrodynamics. Nucl. Phys. A 606, 559 (1996).

45. K.A. Bugaev. Relativistic kinetic equations for finite domains and freeze-out problem. Phys. Rev. Lett. 90, 252301 (2003) and references therein.

46. L.M. Satarov, K.A. Bugaev, I.N. Mishustin. Equation of state and sound velocity of a hadronic gas with a hardcore interaction. Phys. Rev. C 91, 055203 (2015).

47. N. Agrawal et. al. Probing the hadronic phase with resonances of different life-times in $\mathrm{Pb}-\mathrm{Pb}$ collisions with $\mathrm{AL}$ ICE. [ALICE Collaboration] arXiv:1711.02408v1 [hep-ex].

48. L. Adamczyk et. al. [STAR Collaboration] Probing parton dynamics of QCD matter with $\Omega$ and $\phi$ production. Phys. Rev. C 93, 021903 (2016).

49. D. Rischke. Exploring strongly interacting matter at high densities - NICA white paper. Eur. Phys. J. A 52, 267 (2016).
50. P. Senger. Nuclear matter physics at NICA. Eur. Phys. J. A 52, 217 (2016).

51. P. Senger. The compressed baryon matter experiment at FAIR. Nucl. Phys. A 862-863, 139 (2011).

52. T. Ablyazimov et al. Challenges in QCD matter physics the scientific programme of the Compressed Baryon Matter experiment at FAIR. Eur. Phys. J. A 53, 60 (2017).

Received 11.04.18

К.О. Бугаєв, О.І. Іваничъкий,

В.В. Сагун, Е.Г. Ніконов, Г.М. Зінов'єе

РІВНЯННЯ СТАНУ КВАНТОВИХ ГАЗІВ ПОЗА

РАМКАМИ НАБЛИЖЕННЯ ВАН-ДЕР-ВААЛЬСА

$\mathrm{P}$ е $з$ ю м е

Нещодавно запропоноване рівняння стану з індукованим поверхневим натягом узагальнено на випадок квантових газів із взаємодією середнього поля. Для такої моделі знайдено умови самоузгодженості і умови, необхідні для виконання Третього Початку термодинаміки. На відміну від традиційних сподівань показано, що внесення в тиск моделі Ван-дер-Ваальса третього і більш високих віріальних коефіцієнтів газу твердих сфер за низьких температур або порушує Третій Початок термодинаміки, або не дозволяє вийти за рамки наближення Ван-дер-Ваальса. Продемонстровано, що узагальнене рівняння стану з індукованим поверхневим натягом дозволяє уникнути цих проблем і вийти за рамки наближення Ван-дер-Ваальса. Крім цього отримано ефективне віріальне розкладання квантової версї рівняння стану з індукованим натягом і його віріальні коефіцієнти знайдено точно. Явні вирази для справжніх квантових віріальних коефіцієнтів будь-якого порядку цього рівняння стану подано в наближенні низької густини. Обмірковано деякі базові умови на такі моделі, які необхідні для опису властивостей ядерної і адронної матерій. 